\title{
Der Hof Alexanders des Großen als soziales System*
}

\author{
von Gregor Weber
}

\section{Einfübrung}

Der Biograph Plutarch fasst für die Zeit nach Alexanders Zug durch Hyrkanien (Sommer 330 v. Chr.), der zeitlich auf den Tod von Dareios III. folgte, den Habitus des makedonischen Eroberers folgendermaßen zusammen: „Hierauf passte sich Alexander noch mehr in seiner Lebensart den Einheimischen an und suchte andererseits diese den makedonischen Sitten nahezubringen in dem Glauben, dass er durch eine solche Mischung und Gemeinschaft auf göttlichem Wege seine Macht besser begründen werde als durch Gewalt, wenn er sich weit entfernte. ... Da er gewahrte, dass von seinen vertrautesten Freunden Hephaistion sein Verhalten guthieß und die Veränderung der Lebensart mitmachte, Krateros hingegen an den väterlichen Sitten festhielt, so ließ er durch jenen den Verkehr mit den Barbaren, durch diesen den mit den Griechen und Makedonen besorgen, und überhaupt hatte er für den einen die größere Liebe, für den anderen die größere Achtung, und pflegte zu meinen und es immer auszusprechen, Hephaistion sei ein Alexanderfreund, Krateros ein Königsfreund. Daher waren sie feindselig gegeneinander gestimmt und gerieten oft aneinander" (Plutarch, Alexander 47.5.9-11). Die Passage ist in einem Kontext situiert, als Alexander wie auch andere Quellen belegen - begann, die unmittelbare Tradition des Persischen Großkönigs zu adaptieren. ${ }^{1}$ Sie macht deutlich, dass Alexander nicht nur die Reaktionen seiner engsten Umgebung wahrnahm und sie nicht ignorieren konnte, sondern auch, dass die Mitglieder dieser Umgebung auch verschie$d_{e n}$ auf die genannten Veränderungen reagierten; sie standen dabei in einem onfliktiven, auf Konkurrenz basierenden Verhältnis zueinander, das wiederum "on Alexander - so suggeriert die Formulierung bei Plutarch - aktiv befördert Wurde. Direkt im Anschluss an die zitierte Passage wird berichtet, dass Alexander Mühe hatte, beide Freunde, die mit dem Schwert und unter Beteiligung ihrer Gefolgsleute handgemein wurden, dauerhaft zu versöhnen, und dafür in einem

Für Lektüre und Hinweise danke ich Steffen Diefenbach, Hilmar Klinkott, Jürgen Malitz und riten Martin. Eine Kurzfassung dieses Beitrags erscheint in dem von Waldemar Heckel und Larry

Eine Analyse einzelner Maßnahmen bei Fredricksmeyer 2000: 150ff., für den Alexander nicht
inalle herausgegenen Blackwell Companion to Alexander the Great. achfolger des persischen Großkönigs war, sondern einen eigenen Entwurf für eine absolute Mon"On Alber Asien kreierte; zur Ambivalenz Alexanders Persien gegenüber und zur persischen Sicht
(n) Brosius 2003: $171 \mathrm{f}$. und passim; Shapur Shahbazi 2003: 6-19. 
Verhaltensspektrum von Zuneigung bis zur Androhung des Todes, alle Register ziehen' musste. ${ }^{2}$

Dieser Sachverhalt führt zu der Frage, welche strukturelle Relevanz einer solchen Szenerie jenseits der persönlichen Abneigung der Protagonisten zukam. Denn bei diesen handelt es sich um keine geringeren als die Spitzen des neuen makedonisch-persischen Großreiches, mit denen zusammen Alexander seinen Eroberungszug immer weiter vorangetrieben und seine Herrschaft ausgeübt hat. ${ }^{3}$ Diese Herrschaft erforderte freilich eine konkrete Organisation, d.h. das Zentrum, das um Alexander herum gruppiert war, hatte spezifischen personellen und institutionellen Ansprüchen zu genügen, wollte man erfolgreich agieren. ${ }^{4}$ Hierfür steht das Phänomen ,Hof', definiert als das „erweiterte Haus eines Monarchen ", für das die Funktionen Interaktion, Repräsentation und Herrschaft als zentral anzusehen sind. ${ }^{5}$ Dabei waren Alexanders Hof und die an ihm situierte Hofgesellschaft in einer zweifachen Tradition verhaftet: Ausgehend von der Verortung im Kontext der makedonischen Monarchie, die vor allem durch Philipp II. entscheidend geprägt worden war, traten sukzessiv und selektiv persische Elemente hinzu, bis am Lebensende Alexanders etwas Neues entstanden war und sich durch den vergleichsweise langen Aufenthalt in der Residenz von Babylon ein gewisser höfischer Alltag ausgebildet hatte. ${ }^{6}$

Für eine Analyse des Hofes über die Zeitspanne von 336 bis 323 v. Chr. hinweg sind drei Faktoren besonders zu beachten: (1) Wie sich der Hof Alexanders vorwiegend als umherziehendes Heerlager darstellt, das der örtlichen Konstanz entbehrt, so war dieser Hof als soziale Figuration kein statisches, sondern ein dynamisches Gebilde, dessen Entwicklung parallel zur Ausgestaltung der Monarchie verlief. ${ }^{7}$ (2) Eine kritische Würdigung des am Ende erreichten Zustandes fällt schwer, weil aufgrund von Alexanders baldigem Tod und der anschließenden Wirren in der Diadochenzeit diese Form von Hof keine Fortsetzung erfuhr, sich somit über eine Bewährung, Konsolidierung oder gegebenenfalls Modifizierung der Figuration kaum etwas aussagen lässt. ${ }^{8}$ (3) Mit den Quellen für die Alexanderzeit verbindet sich speziell für das Thema ,Hof ' das Problem, dass die

2 Zur Stelle: Bosworth 1980: 7; Völcker-Janssen 1993: 43; Hamilton 1999: 128-131; Müller 2003: 255-259, auch zu den Motiven des Krateros; Whitby 2004: $39 \mathrm{f}$.

3 Zu Hephaistion: Berve 1926: Nr. 357; Heckel 1992: 65-90; Reames-Zimmerman 1999: 89ff. Zu Krateros: Berve 1926: Nr. 446; Heckel 1992: 107-133; Wirth 1993: 348-350. Krateros: Berve 1926: Nr. 446; Heckel 1992: 107-133; Wirth 1993: 348-350.
4 Zu diesen Anforderungen, orientiert an einem typisierten Tagesablauf von Alexander (Plutarch,
Alexander 23): Berve 1926: 11-13. Alexander 23): Berve 1926: 11-13.
5 Die Definition bei A. Winterling, Art. Hof, in: Der Neue Pauly 5 (1998), 661; Weber 1997: passim.
Siehe auch unten S. 232-234.

6 Es darf freilich nicht vergessen werden, dass Babylon nicht als ortsstabiler Endpunkt eines Hofes 6 Es darf freilich nicht vergessen werden, dass Babylon nicht als ortsstabiler Endpunkt eines
gedacht war, denn bereits vor Alexanders Tod wurden noch weitere Unternehmungen, etwa nach
Arabien, geplant. Arabien, geplant.
7 Zur makedonischen Monarchie: Walbank 1984; Hammond 1989: $16 \mathrm{ff}$.; Borza 1990: 236-241;
Hatzopoulos 1996: 37-42.

${ }^{8}$ Scholl 1987: 109. Dass verschiedene Elemente, gerade in zeremonieller Hinsicht (etwa die Pros- 
Primärautoren auf ihre je eigene Weise von der Person Alexanders beeinflusst waren und die Bandbreite seiner Kommunikations- und Interaktionsformen nicht zuletzt nach ihrer persönlichen Involvierung bewerteten. Die Sekundärautoren waren hingegen in Terminologie und Konzeption von den Höfen der späteren hellenistischen Könige und der römischen Principes geprägt; schließlich ergeben sich Schwierigkeiten bei einer angemessenen Erfassung der entsprechenden Verhältnisse im Achaimenidenreich: Diese treten uns nicht nur meist in griechischer Brechung, sondern auch - über zweihundert Jahre hinweg - in einem selektiven Befund gegenüber, der möglicherweise eine zu große Kontinuität suggeriert. ${ }^{9}$

Während sich die Höfe der hellenistischen Könige in den Jahrhunderten nach Alexander eines regen Forschungsinteresses gerade in jüngster Zeit erfreuen, ${ }^{10}$ liegen für den Hof Alexanders nur Detailstudien vor, die einzelne Aspekte der Thematik behandeln. ${ }^{11}$ Deshalb sind im Folgenden zunächst die Konzeption von ,Hof' im Sinne einer Typologie darzulegen und terminologische Fragen zu klären. Dann sollen der Hof Alexanders nicht als geschlossene Einheit, sondern in den beiden genannten Ausprägungen des Anfangs- und Endpunktes entsprechend der Schlüsselkategorien Interaktion, Repräsentation und Herrschaft anaysiert und in einem letzten Abschnitt wesentliche Faktoren der Entwicklung benannt werden. Folgende Fragen sind dabei leitend: Wie war die Hofgesellschaft zusammengesetzt? Welche interaktiven Strukturen lassen sich finden? Welche Mechanismen von Integration und Organisation liefen ab? Wie stellt sich die Relation zwischen erforderlicher Anwesenheit am Hof und dem Ausüben bestimmter Funktionen außerhalb des Hofes dar? Welche Alternativen gab es für prominente Vertreter der jeweiligen Elite zum Hof Alexanders und Wrin liegt die gesamtgesellschaftliche Funktion seines Hofes? Freilich sollte $\mathrm{k} \mathrm{lar}_{\mathrm{r}}$ sein, dass nicht für alle Phasen ein gleiches Informationsniveau zu erzielen ist.

kynese), oder auch die direkte Einbeziehung von Indigenen am Hof (siehe unten Anm. 67), keine

Ortsetzung erfuhren, stellt für sich gesehen auch ein Ergebnis dar.

Bodéüs 1973: passim. Die folgenden beiden Themenbereiche, die ihrerseits eine umfangreiche etaildiskussion hervorgerufen haben, sollen hier ausgespart bleiben: Zum einen die Frage der Vergöttlichung Philipps und besonders Alexanders (zuletzt Wiemer 2005: 163f.); zum anderen die Kontroverse um die Befugnisse der makedonischen Heeresversammlung und deren Formalisierung in A grenzung zum König (Hatzopoulos 1996: $261 \mathrm{ff}$. .).

Habicht 1958, Herman 1997, Weber 1997, Meißner 2000 und Savalli-Lestrade 2003.

Die Personenkreise in Alexanders engster Umgebung und deren Aufgaben behandelt Heckel 1986, 1986a, 2003. Zur Hoforganisation und Hofgesellschaft: Berve 1926: 11-84, bes. $65 \mathrm{f}$., jedoch ohne konzeptionelle Durchdringung; Völcker-Janssen 1993: 7ff.; Einzelaspekte, oft jedoch in anachronistischer Sichtweise, bei Müller 2003: 252-255. 


\section{Hofkonzeption und Terminologie}

Ausgehend von der oben angeführten Definition bezeichnet der Begriff ,Hof zunächst das räumliche Zentrum im Sinne eines erweiterten Hauses (oikos), das ein Herrscher (basileus, monarchos) bewohnte und von dem aus er die politische Regierung und Administration seines Herrschaftsbereiches steuerte. Dieser oikos, in der Regel in einem Zentralort gelegen, konnte insofern architektonisch in besonderer Weise als Palast ausgestaltet sein, als er gesteigerten repräsentativen Bedürfnissen des Herrschers - z. B. für Bankette, Empfänge und kultische Einrichtungen - genügen musste. Hierzu zählten auch die Inszenierung prachtvoller Festlichkeiten und die Demonstration des Reichtums. Als erweitert hat der Hof auch deswegen zu gelten, weil an ihm nicht nur die Familie des Herrschers im engeren Sinne, sondern auch eine gesellschaftliche Elite versammelt war, mit der zusammen der Herrscher agiert hat. Die Beziehung zu dieser Elite hatte entweder informellen Charakter, wenn diese aus Vertrauten des Herrschers (betairoi/Gefährten oder philoi/Freunde) bestand, oder war in Rangstufen mit einem Hoftitelsystem organisiert. ${ }^{12}$ Man kann diese Gruppe, zu der auch das mit der praktischen Organisation betraute Dienstpersonal zu rechnen ist, den engeren oder inneren Hof nennen, wobei das entscheidende Kriterium die echte oder titulare Nähe zum Herrscher darstellte. Davon ist der weitere oder äußere Hof zu unterscheiden, der Gäste, auswärtige Gesandte oder Funktionäre mit zeitweiligem Aufenthalt umfasst. Beide Gruppen der Hofgesellschaft waren nicht hermetisch gegeneinander abgeschlossen, sondern durchlässig. Das Interagieren ihrer Mitglieder, besonders der Familie (Frauen und Geschwister) sowie der Vertrauten des Herrschers, verdient eine genaue Betrachtung. Dies gilt ebenso für die Zusammensetzung der letztgenannten Gruppe im Hinblick auf die geographisch-ethnische Herkunft (Makedonen, Griechen, Perser etc.), auf prosopographische Verflechtungen und auf die Kompetenzen (Militärs, Intellektuelle etc.), welche die Auswahl einzelner Personen bestimmten.

Eine eindeutige, konzeptionell ausgearbeitete griechische Eigenbegrifflichkeit von ,Hof ' und ,Hofgesellschaft' fehlt, statt dessen liegen mehrere Begriffe vor: oikia mit dem Akzent auf dem häuslichen Aspekt, to basileion und ta basileia mit der Betonung des Königs als Besitzer des Hofes sowie aule mit einer lokalen Priorität. Sie sind schwer gegeneinander abzugrenzen, aber sämtlich

12 Für Alexanders Hof kann man trotz mancher begrifflicher Inkonsistenzen bei den antiken Autoren betairoi und philoi synonym gebrauchen: Berve 1926: 30; Corradi 1929: 320f.; Stagakis 1970 90 ff.; Hammond 1989: 54 f.; Borza 1990: 241; Hatzopoulos 1996: 334-336. Eine strikt festgelegte Rangordnung (mit einem dazugehörigen Aufgabenbereich) ist - trotz einiger Formulierungen wie epiphanestatoi (die Herausragendsten') oder pistotatoi (die Vertrautesten') und anders als bei den Militärs (dazu Bosworth 1988: 273-277) - nicht erkennbar, vgl. Berve 1926: 36 f. Auch Hephaistions Sonderstellung unter den hetairoi aufgrund seiner Beziehung zu Alexander (Reames-Zimmerman 1999: 92 f.; Müller 2003: $221 \mathrm{f}$.) liegt auf einer anderen Ebene. 
mit lokaler Konnotation im Sinne von Residenz zu verstehen bzw. bezeichnen verschiedene Teile der Residenz oder betonen unterschiedliche Aspekte von $\mathrm{Zu}$ gänglichkeit und Entzogenheit. ${ }^{13}$ Der Begriff aulè scheint erst für die hellenistischen Höfe aufgekommen zu sein: Er steht nicht nur für den konkreten Königshof, sondern auch für die Hofgesellschaft mit Bediensteten und gesamter Hofhaltung. ${ }^{14}$ Damit eine Residenz als Hof galt, musste die permanente Anwesenheit des Herrschers als zwingende Voraussetzung nicht gegeben sein - im Gegenteil: Sie war in einem Reich wie dem Perserreich mit mehreren Residenzen und einem Großkönig, der saisonalen und militärischen Erfordernissen folgte und dauernd umher zog, nicht möglich. ${ }^{15}$ Jede dieser Residenzen stellte einen ,Hof' dar und hatte zweifellos auch eine Hofhaltung. ${ }^{16}$

Grundsätzlich befand sich eine (besser: die) Zentrale dort, wo sich der Großkönig bzw. - bereits in gleicher Weise von Beginn des Zuges an - Alexander aufhielt. ${ }^{17}$ Dem kriegszugbedingten Charakter des Hofes als mobilem Feldlager entsprach, dass das königliche Zelt (skenēe), das mit allen Erfordernissen für Organisation und Repräsentation ausgestattet war, zum signifikanten Zentrum wurde. Alexander hatte dessen Nutzung, nachdem er den Tross des Dareios mitsamt dem königlichen Harem bei Issos erbeutet hatte, ${ }^{18}$ für sich übernommen und konnte Gäste in großer Zahl bewirten. Die antiken Autoren machen deutlich, dass das Ausmaß an Prunk den für Makedonen üblichen Rahmen deutlich überstieg. Praktische und repräsentative Erfordernisse konnten somit zugleich bedient werden. ${ }^{19}$

Vgl. Funck 1996: 52, der auf den Gebrauch verschiedener Begriffe bei Arrian, Anabasis 7.25 für denselben Sachverhalt, die Königsburg von Babylon, verweist und die Terminologie vor und nach Alexander behandelt.

${ }^{14}$ Funck 1996: 53 f.; Schmitt 2005: $457 \mathrm{f}$. Bezeichnend sind Formulierungen für Höflinge wie hoi peri tèn aulēn (,die am Hof Befindlichen', so Diodor 17.77.4, 17.101.3, 17.110.1) oder aulikoi, was sich nicht bei Diodor und Arrian findet, auch nicht bei Plutarch in der Alexandervita, jedoch z. B. Plutarch, Demetrios 12.8 u. 17.2. Zum Gebrauch bei Polybios, der allem Höfischen kritisch gegenliber stand (Herman 1997: 210): Meißner 2000: 9 f. mit Anm. 27 u. 28.

${ }^{15}$ Bes. Briant 1988; wichtige Aspekte, etwa zur Architektur und zum Verhältnis von Stadt und Palast, bei Boucharlat 2001: 114f., der vom "multi-capital-system of the Achaemenid Empire" spricht und fragt, „how and from which place in his capitals the Great King actually ruled his empire during the construction of the cities and the palaces“. Zu den archäologischen Befunden für Pasargadai, Persepolis, Babylon, Ekbatana und Susa sowie für Aigai und Pella: Nielsen 1994: 31-50 u. 8199; Boucharlat 1997: 219-223; Boucharlat 2001: 115-121.

Funck 1996: $50 \mathrm{f}$.

Dazu Briant 1996: 200-204; Boucharlat 2001: 114: „the impressive royal camp, large and well Organised, was actually the king's seat and played the role of the capital at that moment “. Zum Personellen Umfang, z. B. mit 360 königlichen Konkubinen (Curtius Rufus 3.3.22-23: Briant 1996: 292-295; Llewellyn-Jones 2002: $31 \mathrm{f}$.), insgesamt mehr als 700 Personen: Boucharlat 1997: 219.

Arrian, Anabasis 2.11.10, dazu Briant 1988: $265 \mathrm{f}$.

Arrian, Anabasis 2.12.3-4; 2.20.10 u. passim; Plutarch, Alexander 20.11-13; Diodor 17.35; AtheDaios 12.538c-539d; Curtius Rufus 3.11.23; dazu Briant 1988: 267-269; von Hesberg 1996: 86-88; Gehrke 2003: 93. 


\section{Der Hof Alexanders am Beginn des Persienfeldzugs}

Mit der Sukzession innerhalb der makedonischen Monarchie von Philipp zu Alexander übernahm der neue König den Hof seines Vaters. Als Thronfolger war er mit den Strukturen, ihren Stärken und Schwächen, bestens vertraut. Das Interagieren am Hof war von der rein makedonischen Aristokratie geprägt, die sich in drei Gruppen aufgliedern lässt: Zum einen die Mitglieder der verzweigten Argeadendynastie, zum anderen die Mitglieder verschiedener, überaus mächtiger makedonischer Adelsclans, ${ }^{20}$ schließlich die sogenannten Altersgenossen (syntrophoi) des neuen Königs, die mit ihm zusammen aufgewachsen waren und erzogen wurden. ${ }^{21}$ Für die Interaktion dieser Gruppen untereinander und mit Alexander ist es wichtig festzuhalten, dass sie bereits unter Philipp bestanden bzw. ihm ihre wesentliche Formierung verdankten. ${ }^{22}$

Dass der im Jahre 356 geborene Alexander erfolgreich die Nachfolge seines Vaters antreten konnte, erscheint alles andere als selbstverständlich. Philipp selbst fungierte nämlich ebenso wenig als direkter Thronfolger, sondern $\mathrm{Zu}^{-}$ nächst nur also Vormund seines Neffen Amyntas. Er hatte sich auch nicht auf nur eine Ehe und nur einen Thronfolger festgelegt; vielmehr war er, makedonischem Brauch entsprechend, insgesamt sieben Ehen eingegangen, die alle vor einem politischen Hintergrund zu sehen sind. ${ }^{23}$ Zwar hatte er Alexander bereits über längere Zeit als Nachfolger ,aufgebaut', doch musste seine Hochzeit mit der makedonischen Adligen Kleopatra im Jahre 337 auf Alexander alarmierend gewirkt haben, zumal die Legitimität der Thronfolge von Kleopatras Vormund Attalos angezweifelt wurde. ${ }^{24}$ In die gleiche Richtung wies die Tatsache, dass sich Philipp nicht nur wieder verstärkt seinem Neffen Amyntas zuwandte und diesen mit seiner halbillyrischen Tochter Kynane verheiratete, sondern auch ei- $^{-}$ ne Ehe zwischen Alexanders Schwester Kleopatra und Alexander von Epirus, dem König der Molosser und Bruder von Alexanders Mutter Olympias, arrangierte - zweifellos auch, um letztere zu isolieren. ${ }^{25}$ Diese Vorgänge zeigen einen König, der die Angehörigen seiner Dynastie nach Belieben bzw. nach strategi-

20 Dazu Borza 1990: 237f.; Heckel 2003: 200-203.

${ }^{21}$ Die Jugendfreunde mussten nicht gleich alt sein, dazu Heckel 1986: $301 \mathrm{f}$., und 2003: 203-205, unter Verweis auf Ptolemaios, Nearchos, Harpalos, Erigyios und Laomedon.

${ }^{22}$ Heckels verschiedentlich geäußerte Annahme, es habe Rangabstufungen im Sinne eines, cursus honorum' gegeben (bes. Heckel 1986a), ist nicht belegbar, vgl. Gehrke 2003a: 156.

${ }^{23}$ Satyros bei Athenaios 13.557b-e = Fragmenta Historicorum Graecorum III 161, vgl. Kienast 1973: 30f.; Goukowsky 1991: 60-65; Baynham 1998: 143f.; Ogden 1999: 17 ff.; Carney 2000: 5181; Carney 2003: 228f.; Gehrke 2003: 13 f. u. 19.

${ }^{24}$ Hierzu und zur Pixodaros-Affäre: Hatzopoulos 1986: $290 \mathrm{f}$; Ogden 1999: 20-22; Müller 2003: 27-34. Zu Attalos: Berve 1926: Nr. 182; Heckel 1992: 4 f. Zu Kleopatra und zur gemeinsamen Tochter Europa, die angeblich einige Tage vor Philipps Ermordung geboren wurde: Berve 1926: Nr. ${ }^{34}$; Carney 2000: $77 \mathrm{f}$. Zu den Vorgängen um das Exil von Alexander und Olympias: Carney 2003: 228f.

${ }^{25}$ Diodor 16.91.4-92.1. Zu den Heiratsstrategien Philipps: Carney 2003: 229 u. 231. 
schen Erfordernissen einzusetzen in der Lage war. Selbst Olympias, so ambitioniert sie gewesen sein mag, hatte diesen Agitationen zumindest offen nichts entgegenzusetzen. ${ }^{26}$

Philipps Position schien so gefestigt zu sein, dass ihm auch seitens der makedonischen Aristokratie keine Beschränkungen auferlegt wurden, im Gegenteil: Seine Bemühungen zur Integration hatten sich bewährt. Es war ihm, vermutlich durch entsprechende persische Praxis inspiriert, ${ }^{27}$ definitiv gelungen, die Söhne der Clanchefs aus ganz Makedonien als ,königliche Pagen' (basilikoi paides) an seinen Hof zu ziehen. Diese hatten dort für den König ein vielfältiges Aufgabenspektrum zu erfüllen, sie repräsentierten aber auch ihre Familie am Hof, und nicht zuletzt stellten sie Geiseln dar. Auch dadurch erfuhren die lange nicht in die monarchische Zentralstruktur integrierten Barone aus dem obermakedonischen Bergland eine ,Einbindung ${ }^{6}{ }^{28}$ Philipp hatte eine Gruppe makedonischer Adliger um sich, die auch älter als er sein konnten und hetairoi genannt wurden. ${ }^{29}$ Dazu gehörten u.a. der bereits erwähnte Attalos, dann Parmenion und sein Sohn Philotas, der ,Schwarze Kleitos', Antipatros und Antigonos, der spätere Monophthalmos. ${ }^{30}$ Zwar gelingt es nicht in allen Fällen, die exakte Herkunft dieser Personen, ihre prosopographischen Verbindungen und ihre Karriere zu bestimmen, doch dürfte es sich um diejenigen gehandelt haben, die von Philipp mit den wichtigsten Aufgaben betraut worden waren. Dieser Gruppe wurden auch die sieben königlichen Leibwächter (somatophylakes) entnommen. ${ }^{31}$ Offenkundig verfügten alle über einschlägige militärische Kompetenz und, mit Blick auf die Vorbereitung des Feldzugs gegen die Perser durch Attalos und Parmenion oder auf den weiteren Werdegang des Antipatros, über organisatorisch-administrative Erfahrungen. ${ }^{32}$

Zu Olympias' Rolle unter Philipp: Strasburger 1939: 178; Carney 2003: 230 u. $233 \mathrm{f}$.

Borza 1990: 248f.; Heckel 2003: 205 mit Anm. 38. Zu den grundlegenden Kontakten zwischen Makedonien und dem Perserreich bzw. Mitgliedern der persischen Aristokratie: Heckel 2003: 210 212 am Beispiel des Sisines (Berve 926: Nr. 710); Gehrke 2003a: 143.

Arrian, Anabasis 4.13.1; Diodor 17.65.1; Curtius Rufus 5.1.42 u. 8.6.2-6. Zu ihnen und ihren Aufgaben: Berve 1926: 37-39; Heckel 1986a: 279-285; Hammond 1990: 285 mit der Vorstellung "On einem „system of state education“; Heckel 2003: 205 f.; Gehrke 2003: 18; Vössing 2004: 69 mit Anm. 6.

Griffith in Hammond/Griffith 1979: 395-404; Hammond 1989: 141-148; Hammond 2000: 153 mit Anm. 39 (Belege). Philipp hatte Theopomp, Fragmente Griechischer Historiker (FGrHist) 115 $225 \mathrm{~b}$ (= Athenaios 6.260d-261a) zufolge 800 hetairoi, die mit Land ausgestattet wurden (zum (Oniglichen Landbesitz und entsprechenden Geschenken vgl. Hatzopoulos 1996: 205f. und 335f.). ïr Alexander um 334 wird eine Zahl von 2.800 hetairoi angegeben, was sich aber auf die Hetairenreiterei insgesamt bezieht. Von einem, normalen' Symposion her zu urteilen (siehe unten S. 239 und $250 \mathrm{f}$.) konnten jeweils höchstens 100 hetairoi bewirtet werden.

Zu ihnen und zu ihren Familien: Heckel 1992: 3-64 („The ,Old Guard““),

Zu ihnen, ihren Aufgaben und ihrer Zahl: Corradi 1929: 301-306; Heckel 1986a: 288-293; Ham-

mond 1991: $399 \mathrm{ff}$. , dort auch zum Alter; Heckel 2003: 205-208.

$\chi_{\mathrm{u}}$ Besworth 1988: 5-19; Hammond 1989: 100-119; Borza 1990: 201-225; Errington 1990: 99-102. 
Bekannt ist freilich weder etwas über die Wohnverhältnisse, d.h. in wie weit sie (und ihre Familien) am Hof selbst oder in seinem näheren Umkreis residierten, noch über die Auswahlmechanismen, d. h. auf welche Weise sie in den Kreis um Philipp gelangt waren, doch dürfte Philipp zumindest einige von ihnen von seinem/n Vorgänger/n übernommen haben. Es handelt sich demnach nicht um eine mit dem Herrscher verwandte Aristokratie oder einen Geburtsadel, sondern um eine Gruppe, die dem makedonischen Wertekodex zu genügen hatte und die sich der König aus der Aristokratie zusammen stellen konnte. ${ }^{33}$ Die einzelnen Mitglieder waren, soweit ersichtlich, nicht mit Funktionsstellen ausgestattet, d.h. es bestand keine Koppelung von Zugehörigkeit, konkreten Titeln und festen Aufgaben, sondern der König setzte jeden nach Fähigkeiten und Erfordernissen dafür ein, was es zu erledigen galt. ${ }^{34}$

Wenn nach Philipps Ermordung nicht Amyntas dessen Nachfolge antrat, so verdankte sich dies nicht zum wenigsten der Entscheidung des Antipatros und seiner Familie, Alexander zu unterstützen. ${ }^{35}$ Parmenion und Attalos schlossen sich dieser Option an - letzterer klar gegen seine verwandtschaftlichen Verpflichtungen! ${ }^{36}$ Alexander selbst hat auf dreifache Weise seine Herrschaft gesichert, und dies hängt essentiell mit höfischer Interaktion zusammen: Erstens ließ er diejenigen Mitglieder der Argeadendynastie beseitigen, die ihm gefährlich werden konnten, d. h. potentielle männliche Thronerben. ${ }^{37}$ Damit schloss er aus, dass sich am Hof Faktionen um andere Prätendenten bildeten. Zweitens ging er vor dem Perserfeldzug keine Ehe ein, schon gar nicht mit einer Makedonin, weil auf diese Weise nicht nur eine Familie innerhalb der Aristokratie bevorzugt worden wäre, sondern weil ein in Makedonien zurückgelassener Thronerbe letztlich ein unkalkulierbares Risiko dargestellt hätte. ${ }^{38}$ Drittens ver-

33 Eine wichtige Rolle für die makedonische (und auch persische) Elite, gerade in der Interaktion mit dem König, spielte die erfolgreiche Großwildjagd: Briant 1991: 217-222; Briant 1993: 273ff:; Briant 1996: 242-244 und 309-311; Palagia 2000: 175ff.; Carney 2002, 62-65.

34 Unser Quellenmaterial reicht kaum aus, um ein Bild von den sozialen Verhältnissen am Hof vor Philipp zu zeichnen: Völcker-Janssen 1993: 32-37.

35 Rubinsohn 1977: 416f.; Völcker-Janssen 1993: 37f.; Baynham 1998: 146-148; Heckel 2003: $198 \mathrm{ff}$. Die Ermordung Philipps stellt gewissermaßen ein extremes Mittel der ,Interaktion' dar, das sich in der Folgezeit verstetigen sollte. Jenseits aller Spekulationen über eine Involvierung von Olympias oder gar Alexander selbst lässt sich festhalten, dass die Interessen eines ins Abseits gestellten $\mathrm{Zw} \mathrm{w}^{\mathrm{i}-}$ ges der Dynastie und eines in seiner Ehre verletzten hochrangigen Adligen - Pausanias, der Mörder Philipps, war immerhin hetairos und somatophylax Philipps (Diodor 16.93.3 und 94.4, dazu Philipps, war immerhin hetairos und somatophylax Philipps (Diodor 16.93.3 und 94.4,
McQueen 1995: $176 \mathrm{f}$. und 179f.; zu anderen Quellentraditionen: Berve 1926: Nr. 614) - konvergier
ten (Borza 1999: $65 \mathrm{f}$.).

36 Dies verhinderte nicht die Ermordung des Attalos: Baynham 1998: 146-148; Müller 2003: 40-42. $\mathrm{Zu}$ anderen Fällen von ausbleibender familiärer Loyalität: Heckel 1986: 298-300 und 304f. Zur Gruppe um Parmenion: Heckel 2003: $202 \mathrm{f}$.

37 Dazu zählten sein Cousin Amyntas und die Familie von Alexander v. Lynkestis: Bosworth 1988: 25 f.; Heckel 2003: 199f.; Müller 2003: 34-42. Olympias ließ umgehend ihre Rivalin Kleopatra und deren Tochter aus dem Weg räumen: Carney 2003: 235-237.

38 Antipatros und Parmenion hatten mehrere Töchter; zu deren Ehen: Heckel 1986: 294-296; Bay ${ }^{-1}$ 
traute er sich Personen an, die bereits seinem Vater ergeben waren, und organisierte mit ihnen zusammen die weiteren Feldzüge. Indem er keine herausragenden Gunsterweise verteilte, hielt er die hetairoi in der Balance, so dass sich bestehende Gruppierungen neutralisierten. Dies implizierte, dass die genannte dritte Gruppe der Aristokratie - die mit Alexander gleichaltrigen syntrophoi noch nicht in dem Maße berücksichtigt wurde, wie es später der Fall sein sollte. Alexander entschied sich offenkundig bewusst für die Tradition und Kontinuität, was vor allem aufgrund der Machtkonstellation folgerichtig war. Bereits dem jungen König gelang es bestens, durch den Umgang mit seinen Helfern, was eine entsprechende Auswahl voraussetzt, deren Loyalität und Akzeptanz seiner Herrschaft zu gewinnen. ${ }^{39}$

Ernsthafte Rivalitäten zwischen den Mitgliedern der Hofgesellschaft konnten für den König eine Gefahr bedeuten, wie die eingangs zitierte Passage belegt: Ein zu harmonisches Bild würde in die Irre führen. Man hat vielmehr von einer dauernden Konkurrenz um die Gunst des Königs und von beständigen Faktions(um)bildungen auszugehen. ${ }^{40}$ Dass es kritische Stimmen untereinander und auch dem König gegenüber gab, belegen die Vorgänge um Philipps Ermordung. Auch ist mit opportunistischem Verhalten mancher betairoi zu rechnen, wenn es die eigene Position zu sichern bzw. auszubauen galt. Antipatros' Entscheidung für Alexander dürfte nicht zuletzt von Überlegungen geleitet geweSen sein, welcher der möglichen Prätendenten die besten Überlebens- und Durchsetzungschancen hatte und von wem das intensivste Verpflichtungsgefühl zu erwarten war.

Folgerichtig hat man nach dem Verhältnis des Königs zu den verschiedenen Gruppen an seinem Hof zu fragen. Generell ist davon auszugehen, dass die makedonische Aristokratie miteinander nicht nach Befehl und Gehorsam kommunizierte, im Gegenteil. ${ }^{41}$ Unter den dargelegten Bedingungen kam es aber zu

ham 1998: $151 \mathrm{f}$. Bei Alexander bestand im Unterschied zu seinem Vater (vgl. Carney 1995: 380f. mit dem Verweis auf das Philippeion in Olympia, dazu Huwendiek 1996) kein Interesse an der Präsentation einer dynastischen Figuration: Carney 2003: 230.

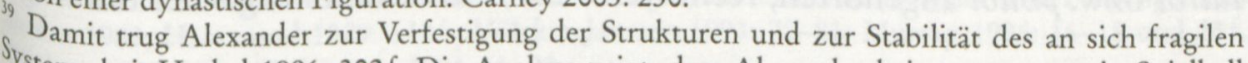
ystems bei: Heckel 1986: 302f. Die Analyse zeigt, dass Alexander keineswegs nur ein Spielball to makedonischen Aristokratie war, wie verschiedentlich behauptet wurde.

Heckel 1986: 305. Das Phänomen der sekundären Gunst, insofern die Gefährten des Königs sie monopolisieren konnten, gab es zwar, bezog sich aber wohl nur auf die Mitglieder der eigenen dekilie. Die Berichte über Philotas (Plutarch, Alexander 48.1-2) zeigen ihn ungemein generös, deklarieren dies aber nicht als vom König abgeleitete Gunst (anders Müller 2003: 251 f., die Alexaners Agieren als gescheiterte Methode der Herrschaftssicherung ansieht). Der Habitus des Harpaos, der sich wie ein eigener Herrscher gerierte, wurde hingegen, wie dessen Flucht nach Griechenand zeigt (Bosworth 1988: 215-220; Wirth 1993: 328-330), als Problem, jedoch nicht als Bedrohung in pfunden.

Dies legen die Zeit vor Philipp und die Schwierigkeiten der Argeaden-Zentrale mit den obermaen len, siehe S. 235. 
einer stärkeren Orientierung auf die Person des Königs: Er gewährte als integrativer Fixpunkt die Gunst, und die damit verbundenen Vorteile waren überaus erstrebenswert. ${ }^{42}$ Allerdings dürfte es für 60 - oder 70jährige hetairoi einen Unterschied gemacht haben, ob sie es mit Philipp oder Alexander zu tun hatten; auch für den jungen König war das Verhältnis zu den annähernd Gleichaltrigen zweifellos ein anderes, das viel stärker auf gemeinsamer Erziehung, Unternehmungen und Freundschaft gründete.

Mitgliedern der Aristokratie, wollten sie ihre Position bewahren oder gar verbessern, stand keine Alternative innerhalb des sozialen Systems zur Verfügung, nur der Rückzug in den eigenen Mikrokosmos. Andere Höfe, etwa in Epirus oder Thrakien, kamen kaum in Frage, allenfalls der Umkreis des persischen Großkönigs; ${ }^{43}$ und in der Welt der griechischen Polis war man als Makedone, zumal aus dem Bergland nahe Illyrien, nicht unbedingt gelitten, selbst wenn an der Ausrichtung des makedonischen Hofes nach Griechenland kein Zweifel bestand. ${ }^{44} \mathrm{Da}$ also die sozialen Chancen am Hof monopolisiert waren, bestand ein reziprokes Interesse an einer Zusammenarbeit - um so mehr, als sich der makedonische Hof in der zweiten Hälfte des 4. Jahrhunderts zu einem echten Machtzentrum entwickelt hatte: Dort waren ebenso Gesandtschaften aus den Poleis, von den persischen Satrapen und vom Großkönig zu finden wie Exilierte aus dem Perserreich. ${ }^{45}$

Makedonischem Habitus entsprach die Zugänglichkeit und die ausgeprägte Vorbildfunktion des Königs: Er musste ein offenes Ohr für die Belange einzelner Bewohner des Landes haben und verfolgte das Kampfgeschehen nicht aus sicherer Distanz, sondern kämpfte an vorderster Front mit. Dennoch hat man davon auszugehen, dass das Leben am Hof und der Zugang zum König bestimmten Regeln unterworfen war; auch ist mit Audienzen zu rechnen, die von einem höfischen Zeremoniell bestimmt gewesen sein dürften. ${ }^{46}$ Mit seiner näheren Umgebung traf sich der König zur Beratung im Thronrat, dem synhedrion; man kann davon ausgehen, dass er für die Bestimmung der Zusammensetzung dieses kaum formalisierten ,Gremiums', dem zweifelsohne die wichtigsten betairoi bzw. philoi angehörten, recht frei war. ${ }^{47} \mathrm{Ob}$ dessen Mitglieder durch ge-

42 Details bei Berve 1926: 34-36, dessen Wertungen man nicht zu teilen braucht.

43 Zum Fall des Amyntas, Sohn des Antiochos: Berve 1926: Nr. 58 (mit Belegen).

44 Baynham 1998: 142f.; Borza 1996 und 1999: $42 \mathrm{f}$. und 47. 44 Baynham 1998: $142 \mathrm{f}$.; Borza 1996 und 1999: $42 \mathrm{f}$. und 47.
45 Plutarch, Alexander 5.1 (Gesandte vom Großkönig); 10.1 (Gesandter des Satrapen Pixodaros);
Diodor 17.2.2 (Gesandte der griechischen Städte).

46 Dass derartige Einrichtungen, ähnlich wie die basilikoi paides (siehe oben Anm. 28), auf persische Vorbilder zurückgehen, ist nicht auszuschließen (Kienast 1973: 28-30; Heckel 1986a: 281 und 1992: 239; anders Briant 1996: 950). Immerhin lebten Mitclieder der persischen Elite, etwa Barsine, ihr Vater Arta Vater Artabazos, Enkel eines persischen Großkönigs, und weitere Familienmitglieder eine Zeis: 117,
am makedonischen Hof im Exil (Diodor 16.52.3; Curtius Rufus 6.5.2-3), dazu McQueen 199:
Carney 2003: 243-245.

47 Diodor 17.16.1f., dazu Corradi 1929: 235-238; Borza 1990: 241; Hatzopoulos 1996: 323-359, mit 
meinsames Tragen purpurner Kleidung erkennbar waren und somit eine Außenwirkung entstand, ist nicht bekannt, aber zu vermuten. ${ }^{48}$ In den Quellen greifbar ist hingegen das gemeinsame Gelage (symposion, potos) des Königs mit seinen Vertrauten, dem aus makedonischer Tradition heraus eine große Bedeutung zukam: ${ }^{49}$ Hier konnten die Teilnehmer - auch wenn zugunsten der ,Erholung die hohe Politik nicht im Zentrum stand - auf informellem Wege Absprachen treffen und vor allem die eigene Position inhaltlich wie sozial definieren; hierin bestand gerade für den König selbst ein probates Mittel, sich Gefolgsleute zu verpflichten und mit diesen von gleich zu gleich umzugehen. ${ }^{50}$

Festhalten lässt sich eine komplexe Interdependenz von König und alter wie junger Aristokratie: Einerseits war der König situationsbedingt auf bestimmte Personengruppen angewiesen, andererseits war er als Herr des Hofes in der Lage, Reichtum, Prestige und Beteiligung an der Herrschaft zu verteilen. ${ }^{51}$ Der Erfolg innerhalb dieses Systems hing vornehmlich von der Person des Königs selbst ab, konkret von seiner Durchsetzungskraft und seinem Charisma.

Die Repräsentation des Königtums fand auf zwei Ebenen statt: Zum einen in der Verkörperung von Monarchie und Monarchen durch Repräsentanten und $\mathrm{Pl}$ atzhalter außerhalb des höfischen Ambiente, etwa an panhellenisch relevanten Orten wie Delos, Olympia oder Delphi, was hier nicht weiter verfolgt werden soll.52 Zum anderen in der Inszenierung der Monarchie am Hof und in der Hauptstadt, etwa in Festen für den Hof selbst und für das außerhöfische Ambiente, bezogen auf die Palastzentren in Pella und Aigai sowie die mobilen Strukturen, wenn sich der König unterwegs befand. Insgesamt ergeben die archäologischen Befunde für diesen Aspekt kaum ein schlüssiges Bild; auch sonst erfahren wir etwa über die Ausgestaltung von Alexanders Wohnquartieren nur venig. ${ }^{53}$ Vor allem scheint man am makedonischen Hof nur für Feste in kleine-

einer umfassenden Analyse aller Detailprobleme, die sich durch den anekdotischen Charakter der Quellen und die anzunehmende zeitliche Entwicklung bei der Valenz des synhedrion ergeben; Heckel 2003: $212 \mathrm{f}$.

${ }_{49}$ Zur purpurnen Kleidung: Errington 1990: 219; Blum 1998: 182-188.

${ }^{49} \mathrm{Dazu}$ und zu verschiedenen Details (Terminologie, Teilnehmerzahl, Wein- und Fleischgenuss): Borza 1983; Hammond 1989: 142f.; Völcker-Janssen 1993: 78-81; Murray 1996: 16-18 und 25 f.; Nielsen 1998: 102-107; Vössing 2004: 66-92.

Dies trifft um so mehr zu, als eine unterschiedliche, in jedem Fall begrenzte Anzahl an Plätzen zur Verfügung stand; hierzu und zur Frage nach den Teilnehmern: Borza 1983: 52-54; Müller 2003:

$254 \mathrm{f}$. Hatzopoulos 1996: 329 hebt mit Recht für entsprechende Gebäude- und Zeltensemble hervor,

$\mathrm{d}_{\text {ass }}$, ,euochiai and government work used to take place in parallel (or jointly).“

${ }^{31}$ Einzelbeobachtungen bei Müller 2003: 250-255, allerdings mit anachronistischen Anleihen im Falle der Konzeption von Monarchie.

S2 Errington 1990: 226f.; verschiedene Beispiele, z. T. in der Datierung unsicher, bei Bringmann/von Steuben 1995: s. v. Philipp II. Nach Plutarch, Alexander 4.9 ließ Philipp seine Wagensiege in Olym$\mathrm{Pi}_{\mathrm{a}}$ auf seine Münzen prägen; zu den entsprechenden Goldstateren und zum weiteren Kontext: Le Rider 1977: 413 mit Pl. 53-65.

Aager (stratópedon): Arrian, Anabasis 1.17.4 u. passim. Quartier in den Häusern von Aspendos: Arian, Anabasis 1.27.2. Allein bei Plutarch (Alexander 9.3) wird von einem Zelt gesprochen, das 
rem Rahmen wie die genannten Symposien gerüstet gewesen zu sein. Bei größerer Dimensionierung musste auf andere Lokalitäten zurückgegriffen werden: So fand Kleopatras Hochzeit mit Alexander von Epirus in dem in unmittelbarer Nachbarschaft zum Palast gelegenen Theater von Aigai statt; ${ }^{54}$ und Diodor berichtet für das Jahr 335/34, dass vor dem Aufbruch nach Persien im makedonischen Dion ein neuntägiges Fest (panegyris) mit Opfern und dramatischen Agonen ausgerichtet wurde, bei der man die Teilnehmer - philoi, hegemones (,Anführer') und presbeis (,Gesandte' ${ }^{c}$ - in einem Festzelt mit einhundert Klinen bewirtet hat. ${ }^{55}$

Bereits unter König Archelaos ist eine Patronage von Dichtern überliefert, vor allem aus dem Bereich der Tragödie; Philipp und Alexander haben diese Tradition fortgesetzt, auch wenn große Namen und konkrete Werke fehlen. ${ }^{56}$ Prestige durch umfassende Förderung von Intellektuellen oder gar von einzelnen Wissenschaftssparten zu erzielen, hat man offenkundig nicht angestrebt. So war der Aufenthalt von Aristoteles am Hof von Pella (343-340 v. Chr.) allein durch die Aufgabe als Erzieher des Thronfolgers bedingt. ${ }^{57}$ Aristoteles' Neffe Kallisthenes nahm - durchaus exzeptionell - als Historiker am Feldzug gegen die Perser teil, ebenso wie verschiedene Philosophen und Dichter, deren genaule Aufgabe jenseits der geistreichen Unterhaltung bei verschiedenen Anlässen unklar bleibt. ${ }^{58}$ Man wird davon ausgehen können, dass sich manch einer von ihnen dem Zug freiwillig oder auf Empfehlung hin anschloss und sich den damit

Alexander im Zusammenhang mit der Schlacht bei Chaironeia bewohnt hatte. Für die spätere Zeit: Hatzopoulos 2001: 193 (Verweis auf Livius 40.6.1 ff.).

54 Zum Theater: Borza 1990: 255 f.; Drougou 1997: 303 f. Zu den Palästen in Aigai und Pella und zu ihrer Größe: von Hesberg 1996: 93; Hatzopoulos 2001: $192 \mathrm{f}$. Gerade die Verhältnisse in Makedonien sind in Oliver Stone's Alexander-Film räumlich überzeugend dargestellt worden: Lane Fox 2004: 74-77. Zum königlichen Erscheinungsbild: von Hesberg 1999: 68. Diodor 16.91.4-95.5 betont, dass Philipp möglichst viele Gäste aus der griechischen Welt einladen wollte, deshalb seine eigenen Gastfreunde aktivierte und seine philoi bat, viele Bekannte von auswärts mitzubringen.

55 Berve 1926: 21; Murray 1996: 17; Hatzopoulos 1996: 329; Aneziri 2003: 57 mit Anm. 213; Vössing 2004: 69f. Zum Festzelt bei Dion nach Diodor 17.16.4: von Hesberg 1996: 86.

56 Weber 1992: 63-70; Weber 1993: 44-51. Plutarch, Alexander 4 berichtet über Aufführungen von Tragödien, Flöten- und Kitharaspieler sowie epische Rezitationen. Plutarch, Alexander 10.2-3 zufolge sandte Alexander den tragischen Schauspieler Thessalos als Gesandten nach Karien, dazu Ane-
ziri 2003: 160 mit Anm. 191.

57 Weber 1992: 67 f.; Rubinsohn 1993: 1307-1311; Scholz 1998: 153-165; Alonso 2000: 23 f.; Carney 2003a: 49-53 und 57-61 (ohne Kenntnis von Weber 1992). Plutarch, Alexander 5 nennt weitere Erzieher, Hofmeister und Pädagogen. Zu einer realistischen Einschätzung der intellektuellen AktiErzieher, Hofmeister und Pädagogen. Zu einer realistischen Einschätzung der intellektuellen Wiemer
vitäten Alexanders, der verschiedene Expeditionen zur Vermehrung des Wissens aussandte: Wies
2005: 177-179.

$58 \mathrm{Zu}$ Agis von Argos, Aischrion, Anaxarchos von Abdera, Anaximenes von Lampsakos, Pranichos oder Pierion, Pyrrhon von Elis und Choirilos von Iasos: Berve 1926: 66-72 (nach Sparten geordnet); Tarn 1971: 55-62; Borza 1983: $51 \mathrm{f}$. Weber 1992: 68f. Carney 2003a: 53-61. Zur Förderung der Historiographie und der makedonischen Tradition: Golan 1988: $100 \mathrm{f}$; Errington 1990: 224f. Zu Alexanders syntrophos Marsyas: Heckel 1980; Rubinsohn 1993: 1313. Zum methodisch sachgemäßen Umgang mit dem meist anekdotischen Quellenmaterial: Brunschwig 1992: $62 \mathrm{f}$. 
verbundenen Strapazen aussetzte, um daraus finanziellen Nutzen und Prestige zu ziehen. ${ }^{59}$

Was den Aspekt der Herrschaft angeht, so stellte der Hof einen organisatorischen Rahmen für deren Ausübung bereit. Dabei spielte nicht nur das synhedrion des Königs mit seinen Gefährten eine Rolle, sondern es gab auch eine königliche Schatzkammer und eine königliche Kanzlei; letztere hatte diverse Aufgaben zu bewältigen, vor allem die Erledigung des privaten und amtlichen Schriftverkehrs, die Zusammenfassung der eingehenden Einzelmeldungen und die Führung der königlichen Tagebücher. ${ }^{60}$ Über die basilikoi paides hinaus ist mit einer Vielzahl von freien und unfreien Bediensteten zu rechnen. ${ }^{61}$ Deren Namen bzw. Funktionsbereiche - etwa als Bäcker und Köche, Bader, Zeltaufbauer - können wir aufgrund der Belegsituation kaum fassen, allenfalls unter der Sammelbezeichnung ,Bedienung' (therapeia). ${ }^{62}$ Sie entsprach den persönlichen Bedürfnissen des Königs und solchen, die für eine erfolgreiche Bewältigung der verschiedenen Außenbeziehungen notwendig waren, ${ }^{63}$ ging es doch nicht allein um Gewaltstrukturen zur Aufrechterhaltung der Herrschaft über die verschiedenen Reichsteile. ${ }^{64}$ Vielmehr war der König gehalten, euergetischen Erfordernissen Rechnung zu tragen: Hier sind vor allem Alexanders Wohltaten einzelnen kleinasiatischen Städten gegenüber zu nennen, die über reine Freiheitsrhetorik und innenpolitische Veränderungen hinaus gingen. Sie beinhalteten z.B. Landschenkungen, Geldzuwendungen und Befreiung von Kontributionen (phoroi), ${ }^{65}$ wobei über die konkrete Abwicklung entsprechen-

${ }^{59}$ Nach Berve 1926: 65 begleiteten Intellektuelle „gleichsam als ,Schlachtenbummler das Heerlager". Eine angebliche Belohnung des Pyrrhon (oder Choirilos) mit 10.000 Goldmünzen für ein Lobgedicht überliefert Sextus Empiricus, adversus mathematicos 282b, dazu Decleva Caizzi 1981: 136 ff.; Brunschwig 1992: 59 f. mit Anm. 2.

${ }^{60}$ Der Schatzkasse stand Harpalos vor; zur Kanzleiarbeit, über deren praktische Seite, etwa für Fragen der Archivierung, kaum etwas bekannt ist: Berve 1926: 42-55; Hammond 1989: 187-192. Die Kanzlei wurde seit 342 von Eumenes v. Kardia geleitet, zu ihm Wirth 1993: 348 f.; Borza 1996: 133-135; Schäfer 2002: 47-51.

Berve 1926: 39-42; Scholl 1987: $109 \mathrm{ff} .$, dort auch zur Schwierigkeit, die basilikoi paides von den Paides (,Sklaven') zu unterscheiden, und zur zeitlichen Entwicklung.

${ }_{63}^{6}$ Eine Übersicht bei Berve 1926: 25; Corradi 1929: $297 \mathrm{f}$.; Scholl 1977: $113 \mathrm{f}$.

${ }^{63}$ Berve 1926: 55-64 macht auch deutlich (59), dass von Alexander selbst wenige Gesandte ausgingen, da er oder Mitglieder seiner Umgebung auch später Verhandlungen nur am eigenen Machtzen${ }_{64}^{t r u m}$ führten. Zum bekannten Beispiel der Gesandten aus Philippi: Hatzopoulos 1997.

Diese spielten bereits angesichts der territorialen Zugewinne unter Philipp eine wichtige Rolle, zu denken ist aber auch an den Schutz des Königs in seinem eigenen Haus und Land: Hammond 1989: $166 \mathrm{ff}$; Errington 1990: 222 betont mit Recht, wie wenig hierzu bekannt ist.

Beispiele bei Hammond 1989: 210-217; Wirth 1993: 72 f.; Alfieri Tonini 2002 (Priene); mit Recht Stark für die einzelnen Städte differenzierend Faraguna 2003: 109-114; Nawotka 2003; Virgilio 2003: $33 \mathrm{f}$. mit Anm. 52. Bedeutsam sind außerdem die Zusendung erbeuteter Rüstungen an Städte und empel in Griechenland (dazu Bringmann/von Steuben 1995: s.v. Alexander d.Gr;; Whitby 2004: 35-37) sowie die angebliche Rückgabe von Kunstgegenständen, die von den Persern geraubt woren waren (Bringmann/von Steuben 1995: Nr. *319). 
der Regelungen bzw. die Involvierung höfischer Strukturen samt Personal kaum etwas bekannt ist.

\section{Der Hof Alexanders in Babylon}

Nach seiner Rückkehr aus dem Osten hielt sich Alexander in den Jahren 324 und 323 wieder im Kernland des Perserreiches, in Susa und Babylon, auf. Hier standen ihm die Paläste der Großkönige mit ihrer gesamten Infrastruktur zur Verfügung, was ein Ende der mobilen Feldlagerstrukturen und neue Möglichkeiten für die herrscherliche Repräsentation implizierte. ${ }^{66}$

Zunächst ist darauf hinzuweisen, dass sich die interaktiven Verhältnisse innerhalb der Hofgesellschaft und deren Zusammensetzung in dreifacher Hinsicht gewandelt hatten: Erstens bestand die engere Umgebung Alexanders nicht mehr allein aus Makedonen, sondern es befanden sich dort jetzt auch Sogdier, Baktrier und vor allem Iraner aus der Aristokratie bzw. der großköniglichen Familie, z.B. Oxyathres, der Bruder des Dareios, und Oxyartes, der Vater der Roxane, unter den hetairoi. ${ }^{67}$ Der ursprünglich rein makedonischen Gruppe wurden nach persischem Vorbild die ,Verwandten' (syngeneis) des Königs zur Seite gestellt. Mit der angezeigten Nähe zum König, ausgedrückt durch die Verleihung des Diadems, waren Aufgaben und Gunsterweise verbunden: ${ }^{8}$ Sie konnten an verschiedene Funktionen außerhalb des Hofes gekoppelt sein, etwa die Betrauung mit einer Satrapie. ${ }^{69}$ Nach welchen Kriterien Alexander diese Personen auswählte, lässt sich kaum sagen. ${ }^{70}$ Es ist auch nichts darüber bekannt, ", how the Persians regarded their role in the corps of Alexander's bodyguards and the

66 Zur persischen und griechischen Begrifflichkeit und deren Bedeutung: Funck 1996: 46-52. Zu den Unterschieden, vor allem mit Blick auf die ,Öffentlichkeit‘ des (Groß-)Königs: von Hesberg 1996: $84 \mathrm{f}$. Zum (ambivalenten) Umgang Alexanders mit den königlichen Städten: Brosius 2003: 181-187. Zu den königlichen Wohnquartieren: Briant 1996: $269 \mathrm{mit}$ dem Verweis auf Diodor 17.71.8; Llewellyn-Jones 2002: $32 \mathrm{f}$.

67 Berve 1926: Nr. 586 und 587; Bosworth 1980: 6 und 12f. (noch zu weiteren Iranern); Collins 2001: 263 f. mit Anm. 27; Brosius 2003: 171. Die einzigen Griechen, die als betairoi bereits vorher in Alexanders unmittelbare Umgebung gelangten, waren Eumenes von Kardia (siehe Anm. 60) - ef fungierte als Kanzleichef und Militärkommandant - und der Kreter Nearchos, zu ihm: E. Badian, Art. Nearchos [2], in: Der Neue Pauly 8 (2000), 777-779. Alexander weicht damit nicht von der Praxis seines Vaters ab: Völcker-Janssen 1993: $39 \mathrm{f}$.

68 Arrian, Anabasis 7.11.2-6, dazu Berve 1926: 37; Vössing 2004: 49 f., Anm. 5, und 86, Anm. 2. Gauger 1977: 157 geht von echten verwandtschaftlichen Bindungen aus, während Briant 1996: $321 \mathrm{f}$. und Jacobs 1996: $275 \mathrm{f}$. und 283 die Ambivalenz in der überlieferten Terminologie betonen und auch und Jacobs 1996: $275 \mathrm{f}$. und 283 die Ambivalenz in der überlieferten Terminologie betonen und
eine titulare Verwendung des Begriffs für möglich erachten. Zum Diadem als Zeichen der syngeneis
Ritter 1965: 7; Jacobs 1996: $277 \mathrm{ff}$.; Stolper 1997.

69 Dies ist jedoch nicht im Sinne einer zwingenden Gleichung Diademträger $=$ Satrapen zu verstehen, so Jacobs 1996: 277. Zur Tradition: Briant 1996: 350-359 („Roi et satrapes“). hen, so Jacobs 1996: 277 . Zur Tradition: Briant 1996: 350-359 („Roi et satrapes“).
70 Wesentlich scheint deren Verhältnis zu seinem Vorgänger gewesen zu sein: Wiesehöfer 1994:
$150 \mathrm{f}$. 
Companions. “71 Immerhin hat Alexander versucht, den Kreis seiner Helfer zu erweitern, Teile der achaimenidischen Elite in das neue Herrschaftsgebilde zu integrieren und somit möglichen Widerstand und Abfallbewegungen zu unterbinden. ${ }^{72}$

Die Bedeutung dieses Schrittes erschöpft sich nicht in der Veränderung des Personenkreises. Entscheidend war vielmehr, dass überhaupt persische Traditionen für Monarchie und Hof übernommen wurden. ${ }^{73}$ Dazu gehört, dass das Verhältnis des Großkönigs zu seiner Umgebung gerade nicht durch die in Makedonien übliche Zugänglichkeit, sondern durch Entzogenheit gekennzeichnet war. ${ }^{74}$ Diese wurde dadurch umgesetzt, dass dem Großkönig bei Auftritten eine exponierte Position zukam und dass sich die persische Elite eines anderen Umgangs mit dem König befleißigte. ${ }^{75}$ Es waren also nicht nur die Titel „im Sinne eines Dienstadels stets mit Ämtern und Funktionen verbunden ", ${ }^{76}$ sondern der Abstand zwischen Großkönig und Elite manifestierte sich besonders in der Proskynese:77 In diesem Ritual einer Kusshand samt Verbeugung, mit deren allgemein verbindlicher Einführung Alexander 327 am Widerstand seiner makedonischen Getreuen scheiterte, sollte die Position des Großkönigs zum Ausdruck gebracht und anerkannt werden. Indem sich Alexander den zeremoniellen Gepflogenheiten der achaimenidischen Tradition gegenüber der Elite unterwarf, versuchte er, in der Selbstdarstellung seine Position zu behaupten bzw. zu stärken. ${ }^{78}$ Bezeichnenderweise lassen sich Angehörige dieser Personengruppe nicht unter den somatophylakes nachweisen: Dies dürfte einerseits darauf zurückZuführen sein, dass bei weitem nicht alle Mitglieder der persischen Elite Alexander anerkannten oder mit ihm kollaborieren wollten; andererseits hätte Alexander eine stärkere Berücksichtigung der Perser ohne weiteres durchsetzen können, wäre aber wohl auf massive Widerstände bei seinen makedonischen Vertrauten gestoßen. Immerhin mussten die Makedonen „the introduction of Persian court offices or titles that were given not to easterners, but to Macedo-

Dies betont zu Recht Brosius 2003: 176.

Zur entsprechenden achaimenidischen Tradition: Högemann 1992: 344-351; Briant 1996: 335-

350. Brosius 2003: 171 mit Anm. 5 sieht Oxyathres und Ochos, den Sohn von Dareios III., als

Geiseln an „whom Alexander could not eliminate, since he regarded himself as the successor to the

murdered Darius III and to the Achaemenid throne. Oxyathres and Ochus were little more than

Prisoners in a gilded cage."

Wiesehöfer 1994: 149-151; Brosius 2003: 179-181.

Briant 1996: 270 f.; Gehrke 2003a: 22.

Funck 1996: 47 (Verweis auf Herodot 1.99). Zu den Rangklassen: Wiesehöfer 1980: 11-14.

A. Mehl, Art. Hoftitel A, in: Der Neue Pauly 5 (1998), $665 \mathrm{f}$.

Zur umfangreichen Diskussion über das Verständnis der Proskynese: Walser 1965: 12 f.; J. Wiese-

höfer, Art. Proskynesis, in: Der Neue Pauly 10 (2001), 443 f.; Vössing 2004: 78-80; Wiemer 2005:

137-140, dort weitere Hinweise.

Wirth 1993: 355-361. 
nians or Greeks “ hinnehmen. ${ }^{79}$ Der indigenen Elite blieb dadurch die unmittelbare und verantwortliche Teilhabe an den Siegen Alexanders verwehrt.

Zweitens hatte sich die makedonische Umgebung Alexanders verändert. Die Männer der alten Garde waren nicht mehr präsent: Antipatros fungierte Zwar als Vertreter Alexanders in Makedonien und Griechenland, hatte jedoch auf die Geschehnisse am Hof und in Alexanders Umgebung keinen unmittelbaren Einfluss. ${ }^{80}$ Dies gilt auch für Antigonos, der als Satrap von Phrygien zurückgelassen worden war. Attalos, Parmenion, Philotas und Kleitos hingegen waren in verschiedenen Zusammenhängen zu Beginn bzw. im weiteren Verlauf des Feldzugs ermordet worden. ${ }^{81}$ Die Gründe hierfür lagen in der Tatsache, dass die drei letztgenannten Alexanders Habitus gegenüber den Persern nicht gutgeheißen hatten. ${ }^{82}$ Aufschlussreich ist die Tatsache, dass diese Helfer Alexanders in ihrem Widerstand keine offenen Fürsprecher hatten, im Gegenteil: Für Alexander bestand die Möglichkeit, sich endgültig von den traditionellen makedonischen Kreisen zu lösen. ${ }^{83}$ Neue Chancen ergaben sich für andere, besonders jüngere betairoi, vor allem für die Personengruppe, die Alexander schon lange nahestand und zu der Koinos, Hephaistion, Leonnatos, Krateros und Perdikkas gehörten, oder für weitere Jugendfreunde Alexanders, etwa Laomedon, Harpalos, Ptolemaios und Nearchos. Aus diesem ,Pool' wurden die sieben Leibwächter (somatophylakes) genommen, wobei die Hintergründe für die jeweilige (Nicht-)Berücksichtigung nicht immer klar sind. ${ }^{84}$ Es handelte sich um eine verschworene Gemeinschaft, die durch unzählige Erlebnisse und gemeinsam bewältigte Strapazen zusammengeschweißt war, deren Mitglieder aber auch unter einem starken Konkurrenzdruck standen und deren Verhältnis von mitunter großer Eifersucht gekennzeichnet war. Die Fähigkeiten und Begabungen dieser Personen lagen - jenseits der emotionalen Bindung - erneut auf militärischem und organisatorischem Gebiet, aber es gab auch Besonderheiten: Für Peukestas ist belegt, dass er im Umgang mit der indigenen Elite und Bevölkerung eine

79 Brosius 2003: $192 \mathrm{f}$. Dies betrifft etwa den eisangeleus („one who announces“), den edeatros (,Vorkoster') und den chiliarchos (,Großwesir', siehe Anm. 100); Belege für die jeweiligen Aufgaben be Collins 2001: $265 \mathrm{f}$.

80 In Makedonien gab es kein Amt des königlichen Stellvertreters mit fest umrissenen Kompetenzen: Carney 2003: $238 \mathrm{f}$., dort auch zu Olympias als Gegengewicht zu Antipatros; zu dessen Befugnissen: Carney 1995: $371 \mathrm{f}$.

81 Alexander hat sich dabei immer der Unterstützung des Heeres und seiner engsten Umgebung versichert: Völcker-Janssen 1993: 43; zur makedonischen Heeresversammlung: Hatzopoulos 1996: 271-273 und passim. Zu Attalos s. Anm. 24; Müller 2003: 55-112 (Parmenion und Philotas), 113133 (Kleitos); Rubinsohn 1977; Carney 1981: 156-160; Whitby 2004: $38 \mathrm{f}$.

${ }_{82}$ Zur Position der makedonischen Aristokratie gegenüber der Orientalisierung der Monarchie: Heckel 2003: 215 und 221f. (Verweis auf Arrian, Anabasis 4.8.4); Müller 2003, $113 \mathrm{f}$.

83 Zu den Umständen der Ermordung und den Hintergründen: Heckel 2003: 215-222. $\mathrm{f}$; Gehrke 84 Berve 1926: 25-30; Rubinsohn 1977: 415; Hammond 1991: $397 \mathrm{ff}$.; Müller 2003: $104 \mathrm{f}$.; Gehrff.; 2003: $66 \mathrm{f}$. Zu den Personengruppen und Konstellationen: Heckel 1992: 57-163, 205-233 und $259 \mathrm{ff}$ Heckel 2003: $119 \mathrm{ff}$. 
besondere (Sprach-)Kompetenz an den Tag legte. ${ }^{85}$ Intellektuelle Fähigkeiten oder gar Prestige in Literatur, Wissenschaft und Kunst waren auch zu dieser Zeit für die hetairoi nicht von sehr großer Bedeutung, wenngleich Eumenes, Ptolemaios und andere bekanntermaßen durchaus Begabungen in diesem Bereich aufweisen konnten. ${ }^{86}$

Insgesamt spielte die persönliche Bekanntschaft mit Alexander, die unbedingte Loyalität ihm gegenüber sowie die Bewährung bei konkreten, vor allem militärischen Aufgaben die entscheidende Rolle. ${ }^{87}$ Allerdings setzte Alexander weitgehend seine Vorstellungen, gerade in personeller Hinsicht, durch; ${ }^{88}$ selbst Hephaistion gegenüber machte er angeblich deutlich, dass er selbst die Quelle jeglicher Gunst darstellte. ${ }^{89}$ Mit zunehmender Größe des Reiches war Alexander auf eine steigende Zahl an zuverlässigen Helfern angewiesen; er hätte diese zunehmend auch dem Kreis der persischen Gefolgschaft entnehmen können. Letztlich blieb also den makedonischen hetairoi, wollten sie nicht ihren Status oder gar ihr Leben verlieren, noch weniger als zuvor eine andere Alternative als mit Alexander zusammen und in seinem Sinne zu agieren: Es erwuchsen ihnen daraus nicht nur Nachteile. ${ }^{90}$

Drittens hat sich Alexander durch die Zeugung von Nachkommen und durch Heiraten anders positioniert als zu Beginn des Zuges. Zwar sollten die Folgen aus dieser Verhaltensänderung erst nach seinem Tod im Kontext des Kampfes um sein Erbe deutlich werden - am Ende stand die Auslöschung der Argeaden-

${ }^{85}$ Peukestas (Berve 1926: Nr. 634; Heckel 1986a: 290f.; Wiesehöfer 1994: 151; Brosius 2003: 177f.) hatte Alexander das Leben gerettet und wurde mit einer eigens geschaffenen achten Stelle als somatophylax geehrt. Arrian, Anabasis 6.28.3-3, 6.30.2-3, dazu Gehrke 2003: 86. Auch Alexanders Jugendfreund Laomedon (Berve 1926: Nr. 464) war des Persischen mächtig und wurde entsprechend eingesetzt (Seibert 2003, 23). Zum Sprachproblem in Alexanders Heer insgesamt: Rochette 1997.

${ }^{86}$ Vgl. Carney 2003a: 60 f., die auch darauf verweist (54), dass Alexander den Maler Apelles und den Bildhauer Lysipp besonders schätzte und ihnen Aufträge zukommen ließ, dazu auch von Hesberg 1998: 205-210.

87 $\mathrm{Zu}$ den Veränderungen bei militärischen Kommandos und den damit verbundenen Aufstiegsmöglichkeiten: Heckel 2003: 209f. Whitby 2004: 37-39 weist darauf hin, dass in der Unterstützung mancher Ideen Alexanders, etwa der Proskynese, für Mitglieder seiner Umgebung die Möglichkeit 8 stand "to advance their own position."

${ }^{8}$ Bekannte Fälle, in denen Alexander von seinen Plänen abrücken musste, waren die von den Soldaten erzwungene Fortsetzung des Zuges über Indien hinaus und die Verbindlichkeit der Prosky-

nese für den makedonischen Teil seiner Umgebung; weitere Beispiele bei Meißner 2000: 13, mit Anm. 39.

Plutarch, Alexander 47.9: „Alexander nannte ihn verrückt und nicht gescheit, wenn er nicht begreife, dass er, wenn man ihm Alexander nähme, ein Nichts sei.“ Zur Isolation Hephaistions: Müller 2003: $258 \mathrm{f}$., derzufolge Hephaistion nicht innerhalb des makedonischen Adelsgeflechts eingebunden war; keine Hinweise auf eine solche Einbindung auch bei Berve 1926: Nr. 357.

Go auch Völcker-Janssen 1993: 40. Wenn Alexander angesichts leerer Kassen von seinen hetairoi Geld forderte (z.B. 1.000 Talente), waren diese Summen zu erbringen, wenngleich Eumenes sich deser Verpflichtung auf trickreiche Weise zu entziehen suchte (100 statt 300 Talente) und von Alea ander - ohne unangenehme Folgen - entlarvt wurde (Plutarch, Eumenes 2.5-6, dazu Hatzopoulos 1996: 335, auch zum historischen Wert der Anekdote). 
dynastie und die Schaffung neuer Dynastien -, doch kam seinem Agieren bereits zu Lebzeiten mehr als nur symbolische Bedeutung zu. Durch die Heirat mit Roxane war zunächst die achaimenidische (und sowieso die makedonische) Elite umgangen, womit sich Alexander eine gewisse Unabhängigkeit bewahrt hatte - und dies offenkundig auch konnte. ${ }^{91}$ Hingegen setzte er durch die im Rahmen der Massenhochzeit von Susa im Jahre 324 erfolgten Heirat mit Stateira, der Tochter von Dareios III., die bereits nach der Schlacht von Issos gefangen genommen worden war, und mit Parysatis, der Tochter von Artaxerxes III., ein anderes Signal: ${ }^{92}$ Es ging jetzt um die Schaffung einer erweiterten Elite für das Reich. Deshalb gerierte sich Alexander nicht mehr (allein) als makedonischer König, wenngleich das Makedonische den Habitus dominierte. Er übernahm eine klare Leitfunktion, und seinen makedonischen Getreuen blieb wohl auch hier keine Alternative zur Mitwirkung. ${ }^{93}$ Dadurch waren auch die persischen ,Verwandten' noch stärker an den König gebunden. Keiner der Frauen kam jedoch eine aktive, auch nach außen demonstrierte politische Rolle zu. ${ }^{94}$ Evident ist auch, dass für Alexander am Ende seines Lebens der Verbindung zur Argeadendynastie sowie damit zusammenhängenden Rücksichten auf den makedonischen Adel eine immer geringere Bedeutung zukam. ${ }^{95}$

91 Dies traf noch mehr für das Verhältnis mit Barsine zu, dem der gemeinsame Sohn Herakles - man beachte den dynastischen Namen - entstammte: Ogden 1999: $42 \mathrm{f}$. und $47 \mathrm{f}$.; Carney 2000: 101-105; Carney 2003: 244f. Zu Alexander und Roxane: Bosworth 1980: 10 f.; Ogden 1999: 43 f.; Carney 2000: 105-107; Carney 2003: $245 \mathrm{f}$.

92 Brosius 1996: 77 f.; Ogden 1999: 44 f.; Carney 2000: 108-111; Carney 2003: 246-248. LlewellynJones 2002: 29f. zufolge kam in der bildlichen und zeremoniellen Selbstdarstellung der persischen Großkönige - anders als in anderen Monarchien des Vorderen Orients - Frauen zwar keinerlei Rolle zu, doch hatte die großkönigliche Familie insgesamt, insbesondere die Mutter des Königs, große Privilegien und Einflussmöglichkeiten; siehe Anm. 94.

93 Gehrke 2003: 87f. Dass sich dies alles der Initiative Alexanders verdankte, wird daraus ersichtlich, dass sich nach seinem Tod die Spur der iranischen Gemahlinnen der hetairoi in der Überlieferung verliert und das Fortbestehen der Ehe von Seleukos und Apama als Ausnahme erscheint; dazu Bosworth 1988: 156-158; Brosius 2003: $176 \mathrm{f}$. Auch die Eliten an den späteren hellenistischen Königshöfen blieben in ihrer Zusammensetzung zunächst rein griechisch-makedonisch (Habicht 1958: 5).

94 Eine solche ist selbst für Olympias und Alexanders Schwester Kleopatra in Makedonien und Epirus kaum greifbar (im Sinne einer prostasia zu hoch eingeschätzt von Hammond 1989: 33 f., dazu Carney 1995: $372 \mathrm{f}$.), doch haben sie - über persönliche Ambitionen hinaus - noch am ehesten von Alexander abgeleitete Macht ausgeübt: Miron 2000: $37 \mathrm{f}$. Carney 2003: 231-234 und 250 f. Nach Plutarch, Alexander 39.12 duldete Alexander nicht, dass seine Mutter sich in Regierungsgeschäfte und militärische Angelegenheiten einmischte, und ertrug auch ihre heftigen Vorwürfe. Zur legendarischen Überformung von Olympias' Agieren, zweifellos im Zusammenhang mit einer retrospektiven Beurteilung durch die antiken Zeitgenossen: Hammond 1989: 34f.; Carney 1995: 383 mit Anm. 38; Miron 2000: 36; Carney 2003: 237-242; Gehrke 2003a: 143. Zu den achaimenidischen Königinnen: Brosius 1996: 105-119. Roxane, die hinsichtlich des Erhalts ihrer Position recht aktiv Königinnen: Brosius 1996: 105-119. Roxane, die hinsichtlich des Erhalts ihrer Position recht Alexan-
gewesen zu sein scheint, ließ unmittelbar nach Alexanders Tod Stateira beseitigen (Plutarch, Ale
der 77.6): Ogden 1999: 46f.; Carney 2003: 246.

95 Es ließe sich darüber spekulieren, ob sich, hätte Alexander länger gelebt, um Frauen und Prätendenten Faktionen gebildet hätten, zumal auch den persischen Großkönigen Polygamie geläufig war 
Fasst man die bisherigen Ausführungen zur inneren Hofgesellschaft zusammen, so wird deutlich, dass es sich um eine künstlich geschaffene, neue Elite gehandelt hat: Hatte Alexander anfangs die Vertrauten seines Vaters bzw. mächtige makedonische Clanchefs akzeptieren müssen, waren diese inzwischen ausgeschaltet und durch Jugendfreunde Alexanders ersetzt. Deren Schicksal war untrennbar mit dem ihres Königs verbunden, mehr noch: Seine Position war so stark, dass er ihnen gegenüber nicht nur seinen Kurs durchsetzen konnte bzw. sie mit ihm zusammen den neuen Kurs forcierten, sondern dass sie, ähnlich wie Soldaten und Kommandeure auf der militärischen Ebene, auch die zunehmende Integration der iranischen Elite hinnehmen mussten. Selbst wenn sich Alexander immer noch vornehmlich mit Makedonen umgab, bestand in der weitergehenden Integration der iranischen Elite und durch die Heiratsverbindungen eine Gefahr für die hetairoi: Die verschiedenen Todesfälle, wie auch immer sie motiviert waren, hatten unmissverständlich deutlich gemacht, wer über das Gewaltmonopol verfügte. ${ }^{96}$ Letztlich war der König immer weniger auf konkrete einzelne Mitglieder seiner Aristokratie angewiesen, zumal deren Bindung nach Makedonien aufgrund der weiten Distanz und der langjährigen Abwesenheit keine reale Bedeutung mehr zukam. ${ }^{97}$ Obwohl man gemeinsam herrschte, sich gemeinsam als hetairoi verstand und die Eliten untereinander und mit dem König selbst verbunden waren, ${ }^{98}$ stellte Alexander die Mitte in einem ,persönlichen Königtum' dar; nicht von ungefähr hat er seinen Freund Hephaistion zum Chiliarchen und somit - in persischer Tradition als Wesir - zum zweiten Mann im heuen Reich ernannt. ${ }^{99}$

Der Zugang zum König, gerade bei Audienzen, war nun weitaus stärker festgelegten Regeln und einem höfischen Zeremoniell unterworfen, das den auf die Person zentrierten Zuschnitt deutlich machte. Vor allem legten die somatophylakes fest, wer zu welcher Zeit zum König vorgelassen wurde. ${ }^{100} \mathrm{Zu}$ diesem Zeremonialisierungsprozess gehört auch, dass Alexander bereits im Jahre 330 nicht nur Teile des Ornats des persischen Großkönigs übernahm, ${ }^{101}$ sondern

(Llewellyn-Jones 2002: $30 \mathrm{f}$.). In der Realität „Olympias and Cleopatra were killed to prevent them $f_{r} \mathrm{~m}$ continuing to exercise power whereas Barsine, Roxane and Stateira were eliminated to prevent others from using them to acquire power" (Carney 2003: 250 f.).

Gehrke 2003: 92: „Bei allen Traditionen und Gefolgschaften, die seine Stellung festigten und egitimierten: Er selbst stand uneingeschränkt darüber, sein Befehl war im Zweifelsfalle das einzige Gesetz. Mit einer Geste konnte er töten und begnadigen, entlassen und aufnehmen, Traditionen

ispektieren oder aufheben."

Völcker-Janssen 1993: $44 \mathrm{f}$.

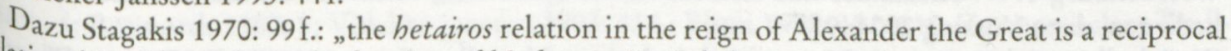

"la ationship; Alexander is the hetairos of his hetairoi"; Gehrke 2003: $92 \mathrm{f}$.

Wirth 1993: 345-347; Collins 2001, 259-262 und 268-274, dort zu Fragen der Terminologie und

tes persischen Vorbilds sowie vor allem zu den vielfältigen Aufgaben; Gehrke 2003: $93 \mathrm{f}$.

Berve 1926: 18-20 u. $27 \mathrm{f}$. In achaimenidischer Zeit gehörte dies zu den Aufgaben des Chiliar-

10, $\mathrm{n}$ : Walser 1965: 13; Collins 2001: 269 und $271 \mathrm{f}$.

Ritter 1965: $31 \mathrm{ff}$. und $41 \mathrm{ff}$., siehe dazu Anm. 111. 
auch dessen Hofstaat mit einer Fülle von Ämtern und Würden sowie mit zahllosen Bediensteten. ${ }^{102}$ So berichtet Diodor: „Als erster richtete er asiatischstämmige Stabträger (rhabdouchous Asiageneis) an seinem Hof ein, dann beorderte er die ausgezeichnetsten der Männer als seine Wachen (doryphorein), unter denen auch der Bruder des Dareios, Oxyathres, war. Dann legte er das persische Diadem um ..." ${ }^{103}$ Unter den Bediensteten spielten Unfreie und besonders Eunuchen, die für den makedonischen Hof nicht belegt sind, ${ }^{104}$ eine große Rolle: In ihrer institutionalisierten Gliederung gehörten sie zum äußeren Erscheinungsbild des Hofzeremoniells; damit wurde auch eine Machtkonzentration in den Händen einzelner Familien verhindert: ${ }^{105}$ Die Disposition des Personals implizierte eine andere Art der Abhängigkeit und des persönlichen Verhältnisses zum König. ${ }^{106}$

Diese Zeremonialisierung wird außerdem im gezielten Einsatz der königlichen Zeltarchitektur deutlich: Um überhaupt dorthin zu gelangen, waren Reihen von Elitetruppen zu passieren. Einer Beschreibung des Phylarchos zufolge machten goldene Platanen und Rebstöcke mit Edelsteinen, unter denen die Perserkönige saßen, ${ }^{107}$ „einen geringeren Eindruck als der Aufwand, der täglich an Alexanders Hof zu allen Gelegenheiten betrieben wurde. Denn dessen Zelt hatte Platz für einhundert Klinen und wurde von fünfzig goldenen Masten gestützt. Oben bildeten die sich darüber spannenden goldenen Himmelsdarstellungen, die mit prächtigen Mustern gearbeitet waren, das Dach. Als erste Reihe standen fünfhundert ,Apfelträger' (mèlophoroi) an der Innenwand darum herum, in purpurne und apfelgelbe Uniformen gekleidet, ${ }^{108}$ dahinter eintausend Bogenschützen, die einen in feuerroter Kleidung, die anderen in scharlachroter, viele hatten aber auch dunkelblaue Umhänge. An deren Spitze standen ferner fünfhundert Makedonen mit silbernen Schilden. In die Mitte des Zeltes wurde

102 Arrian, Anabasis 2.12.5; Diodor 17.38.1; Plutarch, Alexander 20.11 und 21.4; dazu Bosworth 1980: 4 f.; Scholl 1987: 114 f.; Briant 1989; Jacobs 1996: $274 \mathrm{ff}$. Athenaios 13.608a (Brief Parmenions an Alexander) berichtet von 46 Kränzeflechtern, 277 Köchen, 29 Kesselpflegern, 13 Köchen für Milchgerichte, 17 Getränkezubereitern, 70 Weinfilterern und 40 Herstellern von Duftessenzen, dazu Briant 1989, 35; Murray 1996: 19. zu Briant 1989, 35; Murray 1996: 19.
103 Diodor 17.77.4; zu einzelnen Funktionsträgern (auch in anderen Formulierungen): Collins 2001:
262-268.

104 Tougher 2002: 146.

105 Zur Rolle mächtiger Eunuchen am Achaimenidenhof: Briant 1996: 279-288; Llewellyn-Jones 2002: $38 \mathrm{f}$. Zu den Funktionsträgern an der Tafel des Großkönigs: Briant 1996: 304-306; Sancisi2002: $38 \mathrm{f}$. Zu den Funktionsträgern an der Tafel des Großkönigs: Briant 1996: 304-306; San Pagen
Weerdenburg 1997. Alexander ließ sich nicht von Eunuchen, sondern von makedonischen Pän
bedienen, dazu Vössing 2004: 91 .

106 Scholl 1987: 115-118 mit Namen und Aufgaben; Llewellyn-Jones 2002: 33 ff. Negative Wertungen bei Arrian, Anabasis 7.8.3 und Curtius Rufus 6.2.2, dazu Llewellyn-Jones 2002: $22 \mathrm{f}$.

107 Zur Tradition: Briant 1996: 246-249.

108 Sie waren Athenaios 12.514b-c zufolge ausschließlich Perser und wurden aus den zehntausend Unsterblichen' ausgewählt: Bosworth 1980: 9 f.; Scholl 1987: 119f.; Bosworth 1988: 158; Briant 1996: $272 \mathrm{f} . ;$ Blum 1998: 52f. und 61; Brosius 2003: 174. 
ein goldener Thron gestellt, auf dem Alexander saß und die Amtshandlungen vornahm, während die Leibwächter auf allen Seiten dabeistanden. Außerhalb des Zeltes hatte der Elefantentrupp und eintausend Makedonen in makedonischer Tracht Aufstellung genommen, ferner zehntausend Perser und die übrige Menge, die in Purpur gekleidet war; sie belief sich etwa auf fünfhundert Menschen. Ihnen hatte Alexander das Anlegen dieser Kleidung als Auszeichnung verliehen. Da aber die Anzahl seiner Freunde und Gefolgsleute so groß war, wagte es keiner, sich Alexander zu nähern. So hoch war die Wertschätzung, die man seiner Person entgegenbrachte. "109 Aus dieser Passage geht hervor, dass purpurfarbene Kleidung als Auszeichnung und als äußeres Zeichen königlicher Gunst galt. ${ }^{110}$

Von der Kleidung des Königs, die eine Mischung aus makedonischen und persischen Elementen darstellte, berichtet Ephippos: „Zu fast allen anderen $\mathrm{Ge}$ legenheiten hatte er für den täglichen Gebrauch einen Purpurmantel und ein weiß-purpurnes Unterkleid und den breitkrempigen Hut mit dem königlichen Diadem. "111 Derselbe Autor weiß auch von Auftritten Alexanders in seiner engeren Umgebung - wahlweise im Kostüm des Ammon, der Artemis, des Hermes oder des Herakles. Diese Ver-Kleidung ist insofern aufschlussreich, als in der temporären Übernahme einer göttlichen Rolle das Bestreben Alexanders deutlich wird, seine Selbstdarstellung auf einen spezifischen Punkt der Anerkennung zu lenken; ob damit ein expliziter Anspruch auf Göttlichkeit verbunden war, sei dahin gestellt. Wichtig ist die Fortsetzung der Passage, Alexander selbst habe in diesem ,Kostüm ' den Boden mit wertvollem Duftöl und wohlriechendem Wein besprenkelt und für ihn sei wiederum Weihrauch und die anderen Räucheropfer verbrannt worden. Darauf verharrten „alle Anwesenden in Stille und Schweigen vor Furcht; denn er war unberechenbar und schnell bereit zu töten. Denn er schien eine trübsinnige Veranlagung (melancholikós) zu haben." 112 Diese Passage lässt sich - ohne in eine Psychologisierung verfallen zu Wollen - so deuten, dass Alexander durchaus sensibel auf die Reaktion seiner

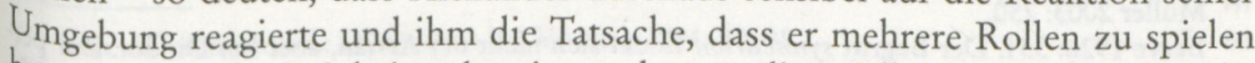
hatte, mehr Schwierigkeiten bereitete, als man dies vielleicht erwarten würde,

${ }^{109}$ Athenaios 12.539d-e = Fragmente Griechischer Historiker (FGrHist) 81 F 41; dazu von Gall 1979: 449 f.; Borza 1983: $46 \mathrm{f}$; von Hesberg 1996: 86; Collins 2001: $264 \mathrm{f}$. Zu berücksichtigen ist freilich, dass Phylarch, ähnlich wie andere Historiker auch, einen dezidiert Alexander-kritischen Kontext evoziert.

${ }^{110}$ Dies ist um so mehr für die engere Umgebung Alexanders anzunehmen: Ritter 1965: 55 mit

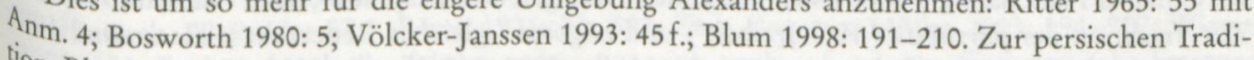
tion: Blum 1998: 49-65.

${ }^{11_{1}}$ Ephippos bei Athenaios 12.537e-f = Fragmente Griechischer Historiker (FGrHist) 126 F 5. Zu Biadem, Kausia und zum kombinierten Gebrauch: Ritter 1965: 55-62; Bosworth 1988: 158. Zur

Bedeutung dieses Aspektes: von Hesberg 1999: 68 f.; Collins 2001, 260f.; Gehrke 2003a: 154.

${ }_{1 / 2}$ Ephippos bei Athenaios 12.538a = Fragmente Griechischer Historiker (FGrHist) 126 F 5. Zum Götterkostüm: Fredricksmeyer 1986; Völcker-Janssen 1993: $46 \mathrm{f}$. 
und dass die Verkleidung mitunter auch ein guter Schutz war. Somit scheint die Aussage des Ephippos, dessen Informationen gemeinhin als zuverlässig angesehen werden, auf eine gute analytische Beobachtung zurückzugehen.

Das äußere Erscheinungsbild war auch für die hetairo $i$ wichtig, denn die antiken Autoren betonen, „dass auch die Gefolgsleute des Alexander in Saus und Braus lebten. Einer von diesen war Hagnon; er trug goldene Nagelköpfe auf seinen Stiefeln. Wenn Kleitos, genannt ,der Weiße', Staatsgeschäfte vorhatte, führte er die Verhandlungen mit denen, die zu ihm kamen, während er auf purpurnen Teppichen umherging. " ${ }^{113}$ Entsprechende Extravaganzen scheinen keine Ausnahme gewesen zu sein, sondern stellten für Alexanders hetairoi eine Möglichkeit dar, ihren Status im Umfeld Alexanders auch nach außen hin sichtbar zu machen. ${ }^{114}$

Über diese offiziellen Auftritte hinaus war Alexander seinen hetairoi weiterhin im synhedrion und vor allem im Symposion verbunden, das als königliches Bankett auch eine lange persische Tradition aufzuweisen hatte. ${ }^{115}$ Besprechungen und Gelage mit der engsten Umgebung fanden bis unmittelbar vor Alexanders Tod statt. Dies erfolgte in einem repräsentativen Rahmen und im Idealfall auch in freier Rede - wenn sie denn der König ertrug. ${ }^{116}$ Ebenso nahm Alexander an Symposien teil, die von seinen hetairoi in ihren jeweiligen Wohnbereichen veranstaltet wurden. ${ }^{117}$ Wichtig blieb die Gleichheit unter den Teilneh-

113 Phylarchos und Agatharchides v. Knidos bei Athenaios $12.539 \mathrm{c}=$ Fragmente Griechischer Historiker (FGrHist) 81 F 41 und 86 F 3, dort auch Bemerkungen zu Perdikkas und Krateros (Zeltbahnen und Sand) sowie zu Leonnatos und Menelaos (Jagdnetze), anders bei Plutarch, Alexander 40.1 (Leonnatos: Sand aus Ägypten; Philotas: Jagdnetze von 100 Stadien Länge), dazu Völcker-Janssen 1993: 113; von Hesberg 1996: 88; Blum 1998: 202; Carney 2002: 62. Die makedonischen Aristokraten führten Plutarch, Alexander 40.1 zufolge eigenes Dienstpersonal mit: Berve 1926: 42; Scholl kraten führten Plutarch, Alexander 40.1 zufolge eigenes Dienstpersonal mit: Berve 1926: 42; Sch 1998 :
1987: 113. Zur Kleidung der hetairoi (z. B. Diodor 17.77.6): Berve 1926: 15-18 und 33; Blum 195.

114 Müller 2003: 250.

115 Berve 1926: 33 f. Der Begriff synhedrion findet sich nicht bei Arrian, aber bei Diodor. Zur persischen Tradition (Athenaios 4.143-146), den dabei konsumierten Gütern und zur politischen Funktion der großköniglichen tryphé: Briant 1988: $262 \mathrm{f}$.; Briant 1989: 36ff.; Briant 1996: $297 \mathrm{ff}$.; Murray 1996: 18-20; Vössing 2004: 38-51. Nielsen 1998: 125 geht davon aus, „that the important thing for 1996: 18-20; Vössing 2004: 38-51. Nielsen 1998: 125 geht davon aus, „that the important thing and
the guests was not to enjoy themselves with conversation in small groups, but to behold the king and
show their respect to him."

116 Ephippos bei Athen. 12.537d = Fragmente Griechischer Historiker (FGrHist) 126 F 4 berichtet für Alexander, „dass für ihn im Park ein goldener Thron und Liegen mit silbernen Füßen standen, auf denen er Platz nahm, wenn er sich mit seinen Gefolgsleuten besprach“, dazu Nielsen 1998: $117 f$. Zur Tradition der parrhēsia (,Redefreiheit') und isēgoria (,gleiches Rederecht ${ }^{\circ}$ ): Hammond 1989: 64 f.; Brunschwig 1992: 67 f.; Murray 1996: 25; Müller 2003: 260; Virgilio 2003: 35 f.

117 Arrian, Anabasis 7.24.4-25.1 berichtet vom einem festlichen Umzug (kömos), der bei Medeios, „damals dem Vertrautesten seiner Hetairen“, stattfand. Der Kontext suggeriert, dass dieser nicht unittelbar in der aulē wohnte (Diodor 17.117.1; Iustin 12.13.7). Nach Aelian, varia historia 3.23 unmittelbar in der aule wohnte (Diodor 17.117.1; Iustin 12.13.7). Nach Aelian, varia historia dian
besaß Bagoas ein Haus bei Babylon, in dem Alexander vor seinem Tod einmal speiste, dazu Bad
1958: 156; Vössing 2004: 87 . 
mern, obwohl persische Elemente, etwa in der Anordnung, eine gegenläufige Tendenz verfolgten. ${ }^{118}$

Der Hof als Mittelpunkt der Herrschaft und als Koordinationszentrum wurde immer wichtiger. ${ }^{119}$ Über die somatophylakes hinaus waren viele der hetairoi nunmehr ständig mit Aufträgen unterwegs. Diese Formen der Interaktion bewirkten eine erhebliche Dynamik der Konkurrenz zwischen den hetairoi, die wiederum auch dafür verantwortlich zeichnete, dass kein Widerstand bzw. keine Verschwörung gegen Alexander von Erfolg gekrönt war. ${ }^{120}$ Auch für diese Phase lässt sich keine Vergabe sekundärer Gunst durch Mitglieder der Umgebung des Königs nachweisen. Aus diesem Grund drohte keine Gefahr für den König, der durchsetzungsfähig war und bei aller Gleichheit den Rang- und Statusunterschied dokumentierte. ${ }^{121}$ Letzteres wird an der Reaktion ersichtlich, die Alexander einem sonst nicht näher bekannten vornehmen Makedonen zuteil werden ließ: „Er war nunmehr aber auch furchtbar und unerbittlich im Bestrafen derer, die sich vergingen. So ließ er den Menandros, einen der Freunde, den er zum Befehlshaber eines festen Platzes ernannt hatte, hinrichten, als er nicht dableiben wollte. " 122 Es verwundert nicht, dass sich ein immer strikteres opportunistisches Verhalten herausbildete, das wiederholt in den griechischen Quellen als Schmeichelei (kolakeia) gegeißelt wurde und sich vor dem Hintergrund des überlieferten Widerstandes einiger weniger um so schärfer abzeichnet: Die Pagenverschwörung und die Vorgänge, die zur Ermordung des Kallisthenes führten, stellten dies jedem nachdrücklich vor Augen. ${ }^{123}$

Eine wichtige Bestätigung für die konstatierten Veränderungen bieten - persönliche Disposition, Profilierungsversuche und Quellenprobleme in Rechnung gestellt - die Reaktionen des im Jahre 324 von seinem Vater Antipatros zu Alexander nach Babylon gesandten Kassandros. Er fand offenbar, gerade mit Blick auf die Proskynese und verschiedene Exzesse, höchst irritierende Zustände vor und hat Plutarch zufolge mit seinem Gelächter Alexander sehr verärgert. Von

${ }^{118}$ Eine differenzierende Diskussion bei Vössing 2004: 90-92.

19 Berve 1926: $28 \mathrm{f}$.

${ }^{120}$ Rubinsohn 1977: $418 f$.; Heckel 2003: 224f.; Müller 2003: $253 \mathrm{f}$. Grundlegend Badian 2000, der auf die in den letzten Lebensmonaten stark ausgeprägte Furcht Alexanders vor Verschwörungen hinweist: „It would be a fitting conclusion to this cycle if he had died as a result of a conspiracy. But our sources are unaccommodating, and we simply do not know“ (89). Zur Rolle von Geheimdienstaktivitäten: Seibert 2003.

${ }^{121}$ Dazu Müller 2003: 251. Die Regelung des Zugangs zum König durch die somatophylakes dürfte im Einvernehmen mit dessen Erwartungen vonstatten gegangen sein.

${ }^{122}$ Plutarch, Alexander 57.3. Berve 1926: Nr. 502. Zum Kontext: Müller 2003: 194-202; Wiemer 2005: 155. Entsprechende Strafaktionen bezogen sich auch auf unbotmäßige persische Satrapen: Bosworth 1988: $240 \mathrm{f}$.

${ }_{123}$ Gehrke 2003: 72; Heckel 2003: 223 f.; Virgilio 2003: $41 \mathrm{f}$. Zur Schmeichelei, etwa am Beispiel des Hagnon v. Teos (Plutarch, de adulatore et amico 24.65d): Berve 1926: Nr. 17. Letztlich ist auch die führende Rolle von Hephaistion bei der Einführung der Proskynese in diesem Sinne zu werten, dazu Whitby 2004: $39 \mathrm{f}$. 
dem zunehmend schlechteren Verhältnis sind bemerkenswerte Nachwirkungen überliefert: „Überhaupt soll sich hiernach eine so heftige und unauslöschliche Furcht in der Seele des Kassandros festgesetzt haben, dass er viele Jahre später ... einmal in Delphi herumging und die Statuen besichtigte, angesichts eines Bildes Alexanders plötzlich vor Schreck schauderte und am ganzen Körper bebte und sich nur langsam erholte; ein solcher Schwindel hatte ihn bei dem Anblick befallen." ${ }^{24}$ Es verwundert auch nicht, dass sich die Gerüchte von einer Vergiftung Alexanders gerade auf die Familie von Antipatros und Kassandros bezogen haben. ${ }^{125}$

Die Repräsentation am Hof blieb auch am Ende von Alexanders Herrschaftszeit wichtig, um so mehr, als diesem Aspekt auch in der Tradition der persischen Monarchie große Bedeutung zukam. Alexander verband hierbei einen „überwiegend am Persischen ausgerichtete[n] Habitus ... mit griechisch-makedonischen Formen der Geselligkeit, mit dem Symposion, mit der Theateraufführung, dem Sportwettkampf.“126 Im Werk der Nikobule heißt es, „dass während des Essens alle möglichen Künstler im Wettstreit bemüht waren, dem König Freude zu bereiten, und dass bei der letzten Mahlzeit Alexander selbst eine bestimmte Szene aus der ,Andromeda' des Euripides auswendig aufsagte und spielte und anschließend mit Leidenschaft den ungemischten Wein trank sowie die anderen dazu veranlasste. "127 Griechische Elemente wurden also nach wie vor gepflegt. Dichter und Philosophen haben weiterhin den Zug begleitet, ohne dass der Überlieferung und den antiken Notizen zufolge qualitätvolle Werke entstanden wären, die den König zum Thema hatten. ${ }^{128}$ Statt dessen traten Konflikte zwischen den Makedonen aus der engeren Hofgesellschaft und allzu willfährigen Intellektuellen, die dann als Schmeichler (kolakes) bezeichnet wurden, ${ }^{129}$ sowie unter den Intellektuellen selbst hervor. ${ }^{130}$ Dass die Aufgabe des Kallisthenes, Alexanders Taten für den Bericht in die Heimat und nach Griechenland aufzubereiten, nach dessen Tod niemand anderem übertragen wurde,

124 Plutarch, Alexander 74.1-6, dazu Berve 1926: Nr. 414; Wirth 1989: $204 \mathrm{f}$. und 213; Landucci Gattinoni 2003.

125 Landucci Gattinoni 2003: 70-72.

126 Gehrke 2003: 93. Zur Repräsentation mit den hetairoi siehe S. $249 \mathrm{f}$.

127 Athenaios $12.537 \mathrm{~d}=$ Fragmente Griechischer Historiker (FGrHist) 127 F 2. Auszugehen ist Jacoby zufolge von einem zeitgenössischen Pamphlet, dessen Autor sich hinter einem Frauennamen versteckt hat. Zu eigenen dichterischen Versuchen Alexanders: Weber 1992: 68, Anm. 343; von Hesberg 1999: 69. Zum Weingenuss: Borza 1983: 47-50.

128 Arrian, Anabasis 1.12.2, dazu Weber 1993: 49f. Zu intensiveren Kontakten mit nicht-grie chischen Intellektuellen scheint es, sieht man von den Gymnosophisten ab, nicht gekommen zu sein. chischen Intellektuellen scheint es, sieht man von den Gymnosophisten ab, nicht gekommen zusans-
${ }^{29}$ Berve 1926: 66 mit dem Verweis auf Curtius Rufus 8.5.7-8. Zum Quellenproblem: Völcker-Jan
sen 1993: 81-84.

130 Zum Konflikt zwischen Kallisthenes und Anaxarchos: Borza 1983: 51 f.; Brunschwig 1992: 66 ff. 130 Zum Konflikt zwischen Kallisthenes und Anaxarchos: Borza 1983: $51 \mathrm{f}$.; Brunschwig 1992: 66
mit einer Analyse von Arrian, Anabasis 4.10.5-12.1; Rubinsohn 1993; Müller 2003: 124-131 und
138-140. 
ist mit den inzwischen veränderten diesbezüglichen Erfordernissen zu erklären. ${ }^{131}$

In Berichten wie aus dem Werk der Nikobule lässt sich die Essenz aus der Wahrnehmung des königlichen Alltags ersehen. Ein herausragendes Ereignis stellte hingegen die fünf Tage dauernde Massenhochzeit zu Susa im Jahre 324 dar, deren Aufwand, wie er in der Beschreibung des Chares von Mytilene zutage tritt, der Funktion, Beteiligte und Zuschauer zu beeindrucken, zweifellos gerecht wurde: „Er ließ zweiundneunzig Hochzeitskammern an demselben Ort errichten. Die Anlage bot Platz für einhundert Klinen. In ihr war jede Kline aus Silber im Wert von zwanzig Minen und mit einem Hochzeitsgewand ausgestattet. Seine eigene Kline jedoch hatte goldene Füße. Er zog aber zum Festmahl auch alle seine privaten Freunde von dort mit hinzu und legte sie sich und den anderen Hochzeitern Angesicht zu Angesicht gegenüber, die restliche Streitmacht, Fußvolk wie auch Flotte, die Gesandtschaften und auch die gerade anwesenden Fremden bewirtete er im Hof. Das Haus aber war prächtig und großzügig mit wertvollen Wandbehängen und Leinentüchern ausgestattet, am Boden mit purpurnen und roten Teppichen, die mit Gold durchwirkt waren. $U_{m}$ dem Zelt einen festen Halt zu geben, stützten es Masten von zwanzig Ellen [fast $9 \mathrm{~m}$ ], die mit Gold und Silber umkleidet und mit Edelsteinen besetzt waren. Im Innenhof befanden sich wertvolle Vorhänge, in die mit Goldfäden Tiermuster eingewirkt und deren Stangen mit Gold und Silber belegt waren. Der Umfang des Hofes betrug vier Stadien [ca. $800 \mathrm{~m}] .{ }^{\text {" } 132}$ Daran schließt sich eine längere Aufzählung der auftretenden Künstler an. ${ }^{133}$ Chares ist nun als Autor und Teilnehmer an Alexanders Feldzug insofern interessant, als er exakt mit dem betraut war, worüber er nach Alexanders Tod auch vornehmlich und sehr positiv schrieb, nämlich mit der Organisation der Audienzen und der zeremoniellen Angelegenheiten (eisangeleus). ${ }^{134}$ Das Fest fand demnach in einer Zeltkonstruktion statt, bei der es sich wahrscheinlich um das genannte Zelt des Großkönigs gehandelt hat: ${ }^{135}$ Entscheidend ist hierbei, dass „die Mobilität und

${ }^{131}$ Golan 1988: 120; Müller 2003: 135 f.; kritisch Rubinsohn 1993: $1302 \mathrm{f}$. und 1319f.; Faraguna 2003: $117 \mathrm{f}$.

${ }^{132}$ Chares bei Athenaios 12.538c-d = Fragmente Griechischer Historiker (FGrHist) 125 F 4; dazu Briant 1988: 268f.; Murray 1996: 19f.; Vössing 2004: 82-84. Zum Ereignis selbst und den damit verbundenen Implikationen: Arrian, Anabasis 7.4.5ff.; Diodor 17.107, dazu Berve 1926: $22 \mathrm{f}$.; Wiemer 2005: $155 \mathrm{f}$.

${ }^{133}$ Die Schwierigkeit der prosopographischen Bestimmung zeigt sich z. B. im Falle des Herakleitos, der - aus Tarent stammend - als Kitharode bei der Hochzeit auftrat und für den - aus Mytilene stammend - ein Namensvetter bekannt ist, der als Zauberkünstler auftrat, vgl. E. Robbins, Herakleitos [2], in: Der Neue Pauly 5 (1998), 385.

${ }^{134}$ Siehe Anm. 47 und 100. Zu Chares: Berve 1926: Nr. 820. Zum palatialen Umfeld von Susa: Boucharlat 2001.

${ }^{135}$ Auch Eumenes ließ auf einen Traum hin ein kostbares Zelt mit einem Thron für Alexander und dessen Herrschaftsinsignien errichten, dazu Hammond 1989a: 219f.; Schäfer 2002: $21 \mathrm{ff}$. (ohne Kenntnis der Arbeiten von Weber 1999 und von Hesberg 1989/1996). 
damit implizit die Vergänglichkeit ... das Einmalige des Festgeschehens “ unterstrichen haben. ${ }^{136} \mathrm{Ob}$ hieran auch bildende Künstler aus Griechenland beteiligt waren, ist schwer zu sagen. ${ }^{137}$

Ein weiteres Ereignis, für das gewaltige Aufwendungen überliefert sind, stellt das Bankett von Opis mit 9.000 Teilnehmern im Sommer 324 dar. Für den vorliegenden Zusammenhang ist nicht die unmittelbare politische Intention Alexanders wichtig, ${ }^{138}$ sondern die Tatsache, dass mit der Anordnung der Personenum ihn herum Makedonen, dann Perser, dann die Elite anderer Völker ${ }^{139}$ - und den religiösen Zeremonien nach griechischer Manier dem repräsentativen $\mathrm{Ab}$ lauf größte Beachtung geschenkt wurde. Die Zielgruppe dieses Aufwands bestand nicht in der inneren Hofgesellschaft, sondern der Hof öffnete sich für die Untertanen besonderer Art, die makedonischen Soldaten: Die Versöhnungsgeste Alexanders kompensierte gewissermaßen den offenkundigen Bedeutungsverlust. Es ging nicht mehr um ein makedonisches Königtum mit makedonischen Elitetruppen, sondern um die Herrschaft über Asien, die notfalls auch mit Indigenen vor Ort aufrecht erhalten werden konnte. ${ }^{140}$

Der repräsentative Aspekt verstärkte sich nochmals während des Aufenthaltes in Ekbatana (Winter 324/23): Alexander richtete - wie auch bereits zuvor ${ }^{141}$ - athletische und musische Wettkämpfe aus, allerdings jetzt mit 3.000 Künstlern und Sportlern, die per Schiff aus Griechenland gekommen waren; außerdem gab er quasi ständig Gelage für seine engste Umgebung. ${ }^{142}$ Vor allem aber sind die 10.000 Talente zu nennen, welche für die Leichenspiele anlässlich der Bestattung des Hephaistion und für dessen monumentales, bei Alexanders Tod noch unvollendetes Grabmal in Babylon aufgewendet worden sein sollen. ${ }^{143}$ Dass dies weder für Ekbatana noch für Makedonien, sondern für Babylon geplant worden war, ist besonders zu beachten: Bedenkt man die kunstvolle Ausgestaltung in

136 von Hesberg 1996: 87 f. mit Anm. 39.

137 Namen und Informationen bei Berve 1926: 76-78; Völcker-Janssen 1993: passim.

138 Dazu und zu Arrian, Anabasis 7.11.8-9: Müller 2003: 212 f.; Vössing 2004: 84-86; Wiemer 2005: $158 \mathrm{f}$.

139 Zum persischen Großkönig „on display“: Llewellyn-Jones 2002: 26.

140 Völcker-Janssen 1993: $43 \mathrm{f}$. Nach Vössing 2004: 85 wurde „hier das persische System in ein griechisches Gewand“ gekleidet. Zur Terminologie und zu Alexanders Verständnis von Asien: Fredricksmeyer 2000: 142-150.

${ }_{141}$ Dazu und bes. zu Arrian, Anabasis 3.1.4, demzufolge bereits Ende 332 bzw. Anfang 331 "auf seine Aufforderung aus Griechenland die berühmtesten Künstler ihres Faches“ zu Agonen nach Memphis kamen: Bloedow 1998.

142 Arrian, Anabasis 7.14.10; Plutarch, Alexander 72.1, weitere Belege, auch für andere Gelegenheiten, bei Borza 1983: 50f.; außerdem Berve 1926: 23 u. 73-76; Weber 1992: 68 mit Anm. 344; Vössing 2004: 81 f. und 86-88.

143 Umstritten ist, ob der Grabbau überhaupt errichtet wurde und ob er mit dem Scheiterhaufen des Hephaistion identisch ist, vgl. die Beschreibung bei Diodor 17.115.1-5, außerdem 18.4.2, Arrian, Anabasis 7.14.8, Plutarch, Alexander 72.3; dazu von Hesberg 1989: 68f.; Völcker-Janssen 1993 100-116; Borchhardt 1993 (bes. mit Tafeln XXXIX-XLIV). Für die Historizität spricht sich mit Recht Palagia 2000: 168-175 aus, anders McKechnie 1995. 
ihrer Wirkung auf die Teilnehmer an der Totenfeier, vor allem auf die hetairoi und die makedonischen Soldaten, so bringt die Situierung an dem Ort innerhalb des Perserreiches, der für Alexander bislang offenkundig am wichtigsten war, zum Ausdruck, dass er dort das Zentrum des neuen Reiches sah und denjenigen unter den hetairoi, der seine Pläne am nachhaltigsten unterstützt hatte, gerade dort exponiert bestatten ließ. ${ }^{144}$ Indem er "durch die Kunst und die Pracht des Baues den Aufwand überbieten“ wollte (Plut. Alex. 72.5), schuf er ein Angebot „an seine Umgebung, sich einem Gefühl der Zusammengehörigkeit und der Überlegenheit hinzugeben, das identitätsbildend wirken und damit die Zustimmung zu seiner Herrschaftskonzeption erleichtern sollte. " 145

Aus den Überlegungen zu den Aufgaben der hetairoi geht hervor, dass sie wesentlich in den organisatorischen Rahmen der Herrschaft integriert waren, sei es als Satrapen, sei es als Militärkommandanten oder im Kontext verschiedenster Detailaufgaben. Zwar kam es nicht zu massiven äußeren Bedrohungen des Reiches, doch war der Übergang von der achaimenidischen auf die makedonische Herrschaft alles andere als einfach. ${ }^{146}$ Hier spielte nicht nur das auf Reziprozität gründende persische System von Wohltaten mit dem Bankett als zentralem Ort für den Gabentausch eine wichtige Rolle, ${ }^{147}$ sondern ebenso der Umgang mit den Poleis in Griechenland und Kleinasien, in deren innere Angelegenheiten Alexander mit dem Dekret über die Rückführung der Verbannten massiv eingriff. ${ }^{148}$

In viel stärkerem Maße als zuvor konnte Alexander auf Verwaltungsstrukturen der Achaimeniden zurückgreifen. Sie waren um so notwendiger, als das Reich mit seiner immensen Ausdehnung einen hohen Organisationsgrad - über das von den Achaimeniden übernommene und den neuen Gegebenheiten angepasste Satrapiensystem hinaus - erforderlich machte. ${ }^{149}$ Dies galt zum einen für die königliche Kanzlei, ${ }^{150}$ zum anderen für die königlichen Schatzhäuser, deren

${ }^{144}$ Völcker-Janssen 1993: 103-105, verweist noch auf den zeitgleich für Philipp II. (wohl in Makedonien) geplanten Grabbau; Palagia 2000: $167 \mathrm{f}$.

145 Völcker-Janssen 1993: 116.

${ }_{146}$ Siehe zu den Widerständen einzelner Satrapen oben Anm. 122. Zur periodischen Erneuerung der Herrschaft über einzelne Reichsteile, die nicht zum wenigsten den Großkönig zum Reisen zwischen Seinen Residenzen bewog: Briant 1988: 270.

${ }_{147} \mathrm{Zu}$ den Wohltätern des persischen Großkönigs und dessen Antwort: Wiesehöfer 1980: 8-11 und 17f.; Jacobs 1996: 274 mit Anm. 28; Briant 1996: 315-319; Vössing 2004: 47: „Der König kaufte keine Lebensmittel, sie wurden ihm vielmehr von überall her ohne Bezahlung geliefert. Das Bankett War insofern nicht nur Symbol, sondern auch direkte Folge seiner Herrschaft." Zu den Kosten: Calmeyer 1980: 58.

${ }_{148}$ Diodor 18.8, dazu Zahrnt 2003; Wiemer 1995: 160-164.

${ }^{149}$ Koch 1990: $217 \mathrm{ff}$., dort auch zum Farnaka (,Hofmarschall ${ }^{\varsigma}$ ) als Spitze der Verwaltung; Klinkott 2000.

${ }^{150}$ Koch 1990: $229 \mathrm{ff}$. mit einer Übersicht über alle an der Hofkanzlei Tätigen. 
,Inhalt' nicht nur der Zurschaustellung des Reichtums, sondern ebenso der Finanzierung neuer Aufgaben, etwa des geplanten Arabienfeldzugs, diente. ${ }^{151}$

\section{Bilanz: Faktoren der Entwicklung}

Die beiden skizzierten Phasen, wie sich Hof und Hofgesellschaft jeweils darstellen, lassen Unterschiede und Gemeinsamkeiten erkennen. Die höfische Interaktion Alexanders mit der Aristokratie ist in der ersten Phase durch ein Verhältnis gekennzeichnet, das unterschiedliche und verschiedenwertige Gruppen integrieren musste; generell gilt, dass Alexander noch in starken Abhängigkeiten von der makedonischen Tradition stand und seine Verhaltensweisen der engeren Umgebung gegenüber von Freundschaft und kluger Berechnung gekennzeichnet waren, die einzelnen Personen durchaus Einfluss gaben, doch auch die eigene Familie nicht als Bedrohungsfaktor konstituierte. Am Ende von Alexanders Leben stellt sich die makedonische Aristokratie um Alexander wesentlich homogener dar, sie musste jedoch eine ,Verstärkung' aus der persischen Elite hinnehmen. Opportunistisches Verhalten nahm zu, zumal alle Widerstände gegen den König weitgehend erfolglos blieben und es zu einer Mitarbeit in seinem Umfeld weder für Griechen und Makedonen, noch für Perser eine Alternative gab. Zahlreiche Berichte über Intrigen am Hof und zwischen den Vertrauten spiegeln jenseits ihres anekdotischen Charakters genau diese interaktiven Verhältnisse wider, die ein sehr hohes Konfliktpotential aufwiesen. ${ }^{152}$ Die Konzentration um den König herum hat zumindest zum Zeitpunkt von Alexanders Tod die Delegation von Herrschaft nicht als strukturellen Schwachpunkt deutlich werden lassen, wie sich denn auch Alexanders Umgebung - sieht man von der partiellen Übernahme achaimenidischer Strukturen einmal ab, zu denen auch die offizielle Position Hephaistions gehörte - nicht in festgelegte Rangklassen aufgliederte. Dies wurde nicht zuletzt dem Umstand geschuldet, dass die Repräsentation von Herrscher und Hof nicht nur der integrativen Selbstinszenierung der herrschenden Gesellschaft in exorbitantem Ausmaß diente, sondern auch die Helfer ihrerseits sich (mit) inszenieren durften, ohne dass damit die eigentliche Führungsrolle angetastet war-der König an sich blieb ohne Alternative.

Diese Repräsentation verlief anfangs in eher bescheidenen makedonischen Bahnen und trug mit einem - soweit für uns noch erkennbar - Mindestmaß an zeremonieller Ausgestaltung besonders den Aspekt der Gleichheit innerhalb der Elite nach außen; für Feste in größerer Dimension musste man auf Hilfskonstruktionen zurückgreifen. Die achaimenidische Tradition erbrachte sowohl in

151 Koch 1990: 235 ff.; Högemann 1992: 276-282 und 340-342; Briant 1996: 820-824.

152 Rubinsohn 1977: 410; Müller 2003: 255 (Verweis auf Ephippos bei Athenaios 12.538a = Fragmente Griechischer Historiker [FGrHist] 126 F 5). 
der Dimension als auch in der Zeremonialisierung eine völlig neue Qualität. Die Darstellung der herrschenden Gesellschaft für die verschiedenen Untertanengruppen spielte eine entscheidende Rolle; der Zurschaustellung immenser Reichtümer in Quantität und Qualität kam dabei große Bedeutung zu. Die in Makedonien inzwischen etablierte Tradition eines gewissen intellektuellen $\mathrm{Zu}$ schnitts der Hofgesellschaft stieß hingegen auf kein persisches Pendant bzw. wurde unter den gegebenen Umständen des Feldzugs stärker institutionalisiert. ${ }^{153}$

$\mathrm{Da}$ am Hof immer die Fäden der administrativen und militärischen Organisation zusammen liefen, diente er auch der Sicherung von Herrschaft; aufgrund des Fehlens mit dem Hof konkurrierender Institutionen gab es hierzu weder eine Alternative noch kam es zu einer Bedrohung. Eine solche erfolgte auch nicht, wie in den späteren hellenistischen Reichen, durch die jeweilige hauptstädtische Bevölkerung. Die Übernahme der entsprechenden achaimenidischen Organisationseinheiten erleichterte die praktische Umsetzung; mit zunehmender Zeitdauer wurden dabei die Verhältnisse in Makedonien und Griechenland immer weniger interessant, sondern von den aktuellen Erfordernissen und weiteren Plänen überlagert.

Die Faktoren, durch welche die Entwicklung von Hof und Hofgesellschaft während des Alexanderzuges entscheidend befördert wurden, lassen sich - um noch einmal zum Anfang zurückzukehren - an der Szene zwischen Hephaistion und Krateros festmachen: Da ist zum einen die Konvergenz von makedonischer und achaimenidischer Tradition in der Monarchie zu nennen. Die letztlich grundlegende Offenheit für das Andere führte in einem bewussten, durchaus auch im Experiment ausgeloteten und vor allem selektiven Rezeptionsprozess $\mathrm{zu}$ etwas Neuem, das ganz individuell auf Alexander zugeschnitten war. Diese individuelle Ausprägung ist nicht zuletzt daran erkennbar, dass Alexanders Nachfolger viele dieser Elemente wieder aufgegeben haben: Die Vereinbarkeit extremer Gegensätze, wie sie Hephaistion und Krateros auf verschiedenen Ebenen repräsentieren, war nur in der Person Alexanders selbst möglich.

Zum anderen ist es Alexander gelungen, die Zusammensetzung seiner Umgebung so zu gestalten, dass sie seinen Zielen förderlich war und sich - und auch dafür stehen Hephaistion und Krateros - mit all ihren Kräften und vor allem auch beachtlichen Fähigkeiten auf verschiedenen Ebenen für seine Sache einsetzte. Dies implizierte mehr als den reinen Austausch einzelner Personen, sondern hatte seinen wesentlichen Grund in der durch Philipp entscheidend beförderten Ausgestaltung der höfischen Strukturen und in einem letzten Aspekt: ${ }^{154}$ Die Eigendynamik des Krieges und der militärische Erfolg, der über Alexan-

${ }^{153}$ Zur persischen Tradition: Briant 1996: 339-342 und 949-950. Dass es in Babylon und anderen Orten des persischen Reiches alte Zentren der Gelehrsamkeit gab (dazu van der Spek 2003), sei hijermit nicht bestritten.

${ }^{154}$ Dazu R. Strootman, Review von Müller 2003, in: Bryn Mawr Classical Review 2004.11.32. 
der hinaus gerade von Helfern wie Hephaistion und Krateros entscheidend befördert wurde, kombiniert mit der andauernden Feldzugssituation, ließ für die Elite eine, geschlossene Gesellschaft' entstehen: ${ }^{155}$ Alle Beteiligten wurden nicht nur sonstiger Bindungen (etwa nach Makedonien) weitestgehend beraubt, sondern waren, auch in ihren administrativen oder militärischen Tätigkeiten, in extremer Weise aufeinander fixiert. Zwar blieb diese herrschende Gesellschaft nicht völlig hermetisch abgeschlossen, im Kern blieb man aber doch unter sich. Der quasi permanente Ausnahmezustand, der auch zum höfischen Alltag wurde, ist deshalb im Zelt des Königs bestens symbolisiert, das erst am Ende von Alexanders Leben von festen Strukturen abgelöst und dennoch weiter verwendet wurde. ${ }^{156}$ Der ,Hof unterwegs' unterschied sich z. B. nicht dadurch von der stationären Residenz, dass der dort anwesende Personenkreis oder der von dort ausgehende Aktionsradius anders war. ${ }^{157}$ Alexanders ,Hof unterwegs ' fehlten hingegen feste Strukturen an der Peripherie: Diese in Mesopotamien zu etablieren, blieb ihm keine Zeit. ${ }^{158}$

\section{Verwendete Literatur:}

T. Alfieri Tonini, Basileus Alexandros, in: P. G. Michelotto (Hg.), Logios aner. Studi di antichità in memoria di Mario Attilio Levi (Mailand, 2002), 1-13.

V. Alonso, La paideia del principe en el tiempo de los Diadocos, in: Ancient History Bulletin 4 (2000), 22-34.

S. Aneziri, Die Vereine der dionysischen Techniten im Kontext der hellenistischen Gesellschaft. Untersuchungen zur Geschichte, Organisation und Wirkung der hellenistischen Technitenvereine (Stuttgart, 2003).

E. Badian, The Eunuch Bagoas, in: Classical Quarterly 8 (1958), 144-157.

E. Badian, Conspiracies, in: A. B. Bosworth-E. J. Baynham (Hgg.), Alexander the Great in Fact and Fiction (Oxford, 2000), 50-95.

E. J. Baynham, Why didn't Alexander Marry Before Leaving Macedonia? Observations

155 Völcker-Janssen 1993: $39 \mathrm{f}$.

156 von Hesberg 1996: 89.

157 Briant 1988: 269: „Ce complex résidentiel mobile n'est pas simplement imposant. Il a valeur symbolique. Le pouvoir n'est pas séparable de la personne du roi: le pouvoir est là où est le roi. Lorsque le roi se déplace, la tente royale - palais mobile - devient le centre d'un pouvoir itinérnat. Tous les hauts officiers de la cour l'accompagnent: le chiliarque, les intendants, les trésoriers, les secrétaires ..."

158 Es wäre überaus reizvoll, danach zu fragen, welche Elemente von Alexanders Hof in späteren monarchischen Konfigurationen weiterentwickelt wurden und welche als rein situationsgebunden anzusehen sind. Ein solcher Vergleich würden den Rahmen dieses Beitrags sprengen und soll deshalb an anderer Stelle erfolgen. Einige Aspekte, etwa zum ,Hof unterwegs', zur Zeremonialisierung in der Spätantike und zum Rückgriff auf Eunuchen, werden in den beiden von A. Winterling herausgegebenen Bänden Zwischen, Haus' und,Staat'. Antike Höfe im Vergleich (München, 1997) und Comitatus. Beiträge zur Erforschung des spätantiken Kaiserhofes (Berlin, 1998) behandelt. 
on Factional Politics at Alexander's Court 336-334 B.C., in: Rheinisches Museum 141 (1998), 141-152.

H. Berve, Das Alexanderreich auf prosopographischer Grundlage. 2 Bde. (München, 1926).

E. F. Bloedow, The Significance of the Greek Athletes and Artists at Memphis in Alexander's Strategy after the Battle of Issus, in: Quaderni Urbinati di Cultura Classica 58 (1998), 129-142.

H. Blum, Purpur als Statussymbol in der griechischen Welt (Bonn, 1998).

R. Bodéüs, Le premier cours occidental. Sur la royauté achéménide, in: Antiquité Classique 42 (1973), 458-472.

J. Borchhardt, Lykische Heroa und die Pyra des Hephaistion in Babylon, in: J. Borchhardt - G. Dobesch (Hgg.), Akten des II. internationalen Lykien-Symposions, Bd. 1 (Wien, 1993), 252-259.

E. N. Borza, The Symposium at Alexander's Court, in: Ancient Macedonia 3 (1983), 4555.

E. N. Borza, In the Shadow of Olympus. The Emergence of Macedon (Princeton, 1990).

E. N. Borza, Ethnicity and Cultural Policy in Alexander's Court, in: Ancient World 22 (1991), 21-25.

E. N. Borza, Greeks and Macedonians in the Age of Alexander. The Source Traditions, in: R. W. Wallace - E. M. Harris (Hgg.), Transitions to Empire. Essays in Greco-Roman History, 360-146 B.C., in Honor of E. Badian (Norman - London, 1996), 122-139.

E. N. Borza, Before Alexander. Constructing Early Macedonia (Claremont, CA, 1999).

A. B. Bosworth, Alexander and the Iranians, in: Journal of Hellenic Studies 100 (1980), 121.

A. B. Bosworth, Conquest and Empire. The Reign of Alexander the Great (Cambridge, 1988).

R. Boucharlat, Camp royal et résidences achéménides, in: Topoi. Orient-Occident, Suppl. 1 (1997), 217-228.

R. Boucharlat, The Palace and the Royal Achaemenid City. Two Case Studies - Pasargadae and Susa, in: I. Nielsen (Hg.), The Royal Palace Institution in the First Millenium BC. Regional Development and Cultural Interchange between East and West (Aarhus, 2001), 113-123.

P. Briant, Conquête territoriale et stratégie idéologique. Alexandre le Grand et l'idéologie monarchique achéménide, in: Actes du colloque international sur l'idéologie monarchique dans l'antiquité (Warschau - Krakau, 1980), 37-83.

P. Briant, Des Achéménides aux rois bellénistiques. Continuités et ruptures (Bilan et propositions), in: P. Briant (Hg.), Rois, tributs et paysans (Paris, 1982), 291-330.

P. Briant, Le nomadisme du Grand Roi, in: Iranica Antiqua 23 (1988), 253-273.

P. Briant, Table du roi, tribut et redistribution chez les Achemenides, in: P. Briant C. Herrenschmidt (Hgg.), Le Tribut dans l'Empire Perse (Paris, 1989), 35-44.

P. Briant, Chasses royales macedoniennes et chasses royales perses. Le théme de la chasse au lion sur La Chasse de Vergina, in: Dialogues d'histoire ancienne 17 (1991), 211-255.

P. Briant, Les chasses d'Alexandre, in: Ancient Macedonia 5/1 (1993), 267-277.

P. Briant, Histoire de l'empire perse de Cyrus à Alexandre (Paris, 1996).

K. Bringmann - H. von Steuben, Schenkungen bellenistischer Herrscher an griechische Städte und Heiligtümer, Bd. 1: Zeugnisse und Kommentare (Berlin, 1995).

M. Brosius, Women in Ancient Persia 559-331 B.C. (Oxford, 1996). 
M. Brosius, Alexander and the Persians, in: J. Roisman (Hg.), Brill's Companion to Alexander the Great (Leiden - Boston, 2003), 169-193.

J. Brunschwig, The Anaxarchus Case. An Essay on Survival, in: Proceedings of the British Academy 82 (1992), 59-88.

P. Calmeyer, Textual Sources for the Interpretation of Achaemenian Palace Decoration, in: Journal of Persian Studies 18 (1980), 55-63.

E. D. Carney, The Death of Clitus, in: Greek, Roman and Byzantine Studies 22 (1981), 149-160.

E. D. Carney, Women and Basileia. Legitimacy and Female Political Action in Macedonia, in: Classical Journal 90 (1995), 369-391.

E. D. Carney, Women and Monarchy in Macedonia (Norman, 2000).

E. D. Carney, Hunting and the Macedonian Elite. Sharing the Rivalry of the Chase (Arrian 4.13.1), in: D. Ogden (Hg.), The Hellenistic World. New Perspectives (London, 2002), 59-80.

E. D. Carney, Women in Alexander's Court, in: J. Roisman (Hg.), Brill's Companion to Alexander the Great (Leiden - Boston, 2003), 227-252.

E. D. Carney, Elite Education and High Culture in Macedonia, in: W. Heckel - L. A. Tritle (Hgg.), Crossroads of History. The Age of Alexander the Great (Claremont, California, 2003), [2003a].

A. W. Collins, The Office of Chiliarch under Alexander and the Successors, in: Phoenix 55 (2001), 259-283.

G. Corradi, Studi ellenistici (Turin, 1929).

F. Decleva Caizzi, Pirrone Testimonianze (Neapel, 1981).

S. Drougou, Das antike Theater von Vergina. Bemerkungen zu Gestalt und Funktion in der antiken Hauptstadt Makedoniens, in: Mitteilungen des Deutschen Archäologischen Instituts (Athen) 112 (1997), 281-305.

R. M. Errington, A History of Macedonia (Berkeley, 1990).

M. Faraguna, Alexander and the Greeks, in: J. Roisman (Hg.), Brill's Companion to Alexander the Great (Leiden - Boston, 2003), 99-130.

E. A. Fredricksmeyer, Alexander the Great and the Macedonian Kausia, in: Transactions and Proceedings of the Philological Association 116 (1986), 215-227.

E. Fredricksmeyer, Alexander the Great and the Kingship of Asia, in: A. B. BosworthE. J. Baynham (Hgg.), Alexander the Great in Fact and Fiction (Oxford, 2000), 136166.

B. Funck, Beobachtungen zum Begriff des Herrscherpalastes und seiner machtpolitischen Funktion im bellenistischen Raum. Prolegomena zur Typologie der hellenistischen Herrschaftssprache, in: W. Hoepfner - G. Brands (Hgg.), Basileia. Die Paläste der bellenistischen Könige (Mainz, 1996), 44-55.

H. von Gall, Das Zelt des Xerxes und seine Rolle als persischer Raumtyp in Griechenland, in: Gymnasium 86 (1979), 444-462.

J.-D. Gauger, Zu einem offenen Problem des hellenistischen Hoftitelsystems. Ein persischer Ehrentitel , syngenés'?, in: A. Lippold - N. Himmelmann-Wildschütz (Hgg.), Bonner Festgabe Johannes Straub (Bonn, 1977), 137-158.

H.-J. Gehrke, Alexander der Große (München, $\left.{ }^{3} 2003\right)$.

H.-J. Gehrke, Geschichte des Hellenismus (München, ${ }^{3} 2003$ ) [Gehrke 2003a].

D. Golan, The Fate of a Court Historian. Callisthenes, in: Athenaeum 66 (1988), 99-120.

P. Goukowsky, Les maisons princières de Macédoine de Perdiccas II à Philippe II, in 
P. Goukowski - C. Brixhe (Hgg.), Hellènika Symmikta. Histoire, archéologie, épigraphie (Nancy, 1991), 43-66.

Chr. Habicht, Die herrschende Gesellschaft in den hellenistischen Monarchien, in: Vierteljahrschrift für Sozial- und Wirtschaftsgeschichte 45 (1958), 1-16.

J. R. Hamilton, Plutarch. Alexander (London, ${ }^{21999) . ~}$

N. G. L. Hammond, The Macedonian State. Origins, Institutions, and History (Oxford, 1989).

N. G. L. Hammond, Arms and the King. The Insignia of Alexander the Great, in: Phoenix 43 (1989), 217-224 [Hammond 1989a].

N. G. L. Hammond, Royal Pages, Personal Pages, and Boys Trained in the Macedonian Manner during the Period of the Temenid Monarchy, in: Historia 39 (1990), 261-290.

N. G. L. Hammond, The Various Guards of Philip II and Alexander III, in: Historia 40 (1991), 396-417.

N. G. L. Hammond, The Continuity of Macedonian Institutions and the Macedonian Kingdoms of the Hellenistic Era, in: Historia 49 (2000), 141-160.

N. G. L. Hammond - G. T. Griffith, A History of Macedonia, Bd. 2: 550-336 B.C. (Oxford, 1979).

M. B. Hatzopoulos, Succession and Regency in Classical Macedonia, in: Ancient Macedonia 4 (1986), 279-292.

M. B. Hatzopoulos, Macedonian Institutions under the Kings, Bd. 1: A Historical and Epigraphic Study (Athen - Paris, 1996).

M. B. Hatzopoulos, Alexandre en Perse: la revanche et l'empire, in: Zeitschrift für Papyrologie und Epigraphik 116 (1997), 41-52.

M. B. Hatzopoulos, Macedonian Palaces. Where King and City Meet, in: I. Nielsen (Hg.), The Royal Palace Institution in the First Millenium BC. Regional Development and Cultural Interchange Between East and West (Aarhus, 2001), 189-199.

W. Heckel, Marsyas of Pella, Historian of Macedon, in: Hermes 108 (1980), 444-452.

W. Heckel, The ,Boybood Friends' of Alexander the Great, in: Emerita 3 (1985), 285-289.

W. Heckel, Factions and Politics in the Reign of Alexander the Great, in: Ancient Macedonia 4 (1986), 293-305.

W. Heckel, Somatophylakia. A Macedonian Cursus Honorum, in: Phoenix 40 (1986), 279-294 [Heckel 1986a].

W. Heckel, The Marshalls of Alexander's Empire (London - New York, 1992).

W. Heckel, King and,Companions. Observations on the Nature of Power in the Reign of Alexander, in: J. Roisman (Hg.), Brill's Companion to Alexander the Great (LeidenBoston, 2003), 197-225.

G. Herman, The Court Society of the Hellenistic Age, in: P. Cartledge (Hg.), Hellenistic Constructs. Essays in Culture, History and Historiography (Berkeley, 1997), 199-224.

H. von Hesberg, Temporäre Bilder oder: Die Grenzen der Kunst, in: Jahrbuch des deutschen archäologischen Instituts 104 (1989), 61-82.

H. von Hesberg, Privatheit und Öffentlichkeit der frühhellenistischen Hofarchitektur, in: W. Hoepfner - G. Brands (Hgg.), Basileia. Die Paläste der bellenistischen Könige (Mainz, 1996), 84-96.

H. von Hesberg, Riti e produzione artistica delle corti ellenistiche, in: S. Settis (Hg.), I Greci. Storia Cultura Arte Società, Bd. 2: Una storia greca. III. Trasformazioni (Turin, 1998), 177-214.

H. von Hesberg, The King on Stage, in: B. Bergmann - C. Kondoleon (Hgg.), The Art of Spectacle (Washington, 1999), 65-76. 
P. Högemann, Das alte Vorderasien und die Achämeniden. Ein Beitrag zur HerodotAnalyse (Wiesbaden, 1992).

J. Huwendiek, Zur Interpretation des Philippeion in Olympia, in: Boreas 19 (1996), 155159.

B. Jacobs, Die ,Verwandten des Königs' und die, Nachkommen der Verschwörer. Überlegungen zu Titeln, Ämtern und Insignien am Achämenidenhof, in: F. Blakolmer et al. (Hgg.), Fremde Zeiten. Festschrift für Jürgen Borchhardt zum 60. Geburtstag am 25. Februar 1995 (Wien, 1996), 273-284.

D. Kienast, Philipp II. von Makedonien und das Reich der Achaimeniden (München, 1973).

H. Klinkott, Die Satrapienregister der Alexander- und Diadochenzeit (Stuttgart, 2000).

H. Koch, Verwaltung und Wirtschaft im persischen Kernland zur Zeit der Achämeniden (Wiesbaden, 1990).

F. Landucci Gattinoni, L'arte del potere. Vita e opere di Cassandro di Macedonia (Stuttgart, 2003).

F. Landucci Gattinoni, Plutarco e un modello di eroe negativo: il caso di Cassandro figlio di Antipatro, in: A. Barzanò et al. (Hgg.), Modelli Eroici dall'Antichità alla Cultura Europea (Rom, 2003), 169-184 [2003a].

R. Lane Fox, The Making of Alexander (Oxford - London, 2004).

G. Le Rider, Le monnayage d'argent et d'or de Philippe II frappé en Macédoine de 359 à 294 (Paris, 1977).

L. Llewellyn-Jones, Eunuchs and the Royal Harem in Achaemenid Persia, in: S. Tougher (Hg.), Eunuchs in Antiquity and Beyond (London, 2002), 19-50.

P. McKechnie, Diodorus Siculus and Hephaestion's Pyre, in: Classical Quarterly 45 (1995), 418-432.

E. I. McQueen, Diodorus Siculus. The Reign of Philipp II. The Greek and Macedonian Narrative from Book XVI. A Companion (London, 1995).

B. Meißner, Hofmann und Herrscher. Was es für die Griechen hieß, Freund eines Königs

zu sein, in: Archiv für Kulturgeschichte 82 (2000), 1-36.
D. Miron, Transmitters and Representatives of Power. Royal Women in Ancient Macedonia, in: Ancient Society 30 (2000), 35-52.

S. Müller, Maßnabmen bei der Herrschaftssicherung gegenüber der makedonischen OPposition bei Alexander dem Großen (Frankfurt/M., 2003).

O. Murray, Hellenistic Royal Symposia, in: P. Bilde et al. (Hgg.), Aspects of Hellenistic Kingship (Aarhus, 1996), 15-26.

K. Nawotka, Freedom of Greek Cities in Asia Minor in the Age of Alexander the Great, in: Klio 85 (2003), 15-41.

I. Nielsen, Hellenistic Palaces. Tradition and Renewal (Aarhus, 1994).

I. Nielsen, Royal Banquets. The Development of Royal Banquets and Banqueting Halls from Alexander to the Tetrarchs, in: I. Nielsen-H. S. Nielsen (Hgg.), Meals in a Social Context. Aspects of the Communal Meal in the Hellenistic and Roman World (Aarhus, 1998), 102-133.

D. Ogden, Polygamy, Prostitutes and Death. The Hellenistic Dynasties (London, 1999).

O. Palagia, Hephaestion's Pyre and the Royal Hunt of Alexander, in: A. B. BosworthE. J. Baynham (Hgg.), Alexander the Great in Fact and Fiction (Oxford, 2000), 167206.

J. Reames-Zimmerman, An Atypical Affair? Alexander the Great, Hephaistion Amyntoros and the Nature of Their Relationship, in: Ancient History Bulletin 13 (1999), 81-96. 
H.-W. Ritter, Diadem und Königsherrschaft. Untersuchungen zu Zeremonien und Rechtsgrundlagen des Herrschaftsantritts bei den Persern, bei Alexander dem Großen und im Hellenismus (München - Berlin, 1965).

B. Rochette, Les armées d'Alexandre le Grand et les langues étrangères, in: L'Antiquité Classique 66 (1997), 311-318.

W. Z. Rubinsohn, The ,Philotas Affair - a Reconsideration, in: Ancient Macedonia 2 (1977), 409-420.

W.Z. Rubinsohn, The Philosopher at Court. Intellectuals and Politics in the Time of Alexander the Great, in: Ancient Macedonia 5/2 (1993), 1301-1327.

H. Sancisi-Weerdenburg, Crumbs fom the Royal Table. Foodnotes on Briant (297-306), in: Topoi. Orient-Occident, Suppl. 1 (1997), 333-345.

I. Savalli-Lestrade, La place des reines à la cour et dans le royaume à l'époque hellénistique, in: R. Frei-Stolba et al. (Hgg.), Les femmes antiques entre sphère privée et sphère publique (Bern, 2003), 59-76.

C. Schäfer, Eumenes von Kardia und der Kampf um die Macht im Alexanderreich (Frankfurt/M., 2002).

H. H. Schmitt, Art. Hof, in: ders. - E. Vogt (Hgg.), Lexikon des Hellenismus (Wiesbaden, 2005), 457-462.

R. Scholl, Alexander der Große und die Sklaverei am Hofe, in: Klio 69 (1987), 108-121.

P. Scholz, Der Philosoph und die Politik. Die Ausbildung der philosophischen Lebensform und die Entwicklung des Verbältnisses von Philosophie und Politik im 4. und 3. Jh. v. Chr. (Stuttgart, 1998).

J. Seibert, Der Geheimdienst Alexanders des Großen (336-323 v. Chr.), in: W. Krieger (Hg.), Geheimdienste in der Weltgeschichte. Spionage und verdeckte Aktionen von der Antike bis zur Gegenwart (München, 2003), 19-29.

A. Shapur Shahbazi, Irano-Hellenic Notes. 3. Iranians and Alexander, in: American Journal of Ancient History n.s. 2.1 (2003), 5-38.

R. J. van der Spek, Darius III, Alexander the Great and Babylonian Scholarship, in: W. Henkelman - A. Kuhrt (Hgg.), A Persian Perspective. Essays in Memory of Heleen Sancisi-Weerdenburg (Leiden, 2003), 289-346.

G. S. Stagakis, Observations on the ,hetairoi" of Alexander the Great, in: Archaia Makedonia 1 (1970), 86-102.

M. W. Stolper, Flogging and Plucking, in: Topoi. Orient-Occident, Suppl. 1 (1997), 347350.

H. Strasburger, s. v. Olympias 5, in: Realencyclopädie der Classischen Altertumswissenschaften XVIII, 1 (1939), 177-182.

W. W. Tarn, Alexander the Great, 2 Bde. (Boston, 1971).

S. F. Tougher, In or out? Origins of Court Eunuchs, in: S. Tougher (Hg.), Eunuchs in Antiquity and Beyond (London, 2002), 143-160.

B. Virgilio, Lancia, diadema e porpora. Il re e la regalità ellenistica (Pisa - Rom, 2003).

W. Völcker-Janssen, Kunst und Gesellschaft an den Höfen Alexanders des Großen und seiner Nachfolger (München, 1993).

K. Vössing, Mensa Regia. Das Bankett beim hellenistischen König und beim römischen Kaiser (München - Leipzig, 2004).

F. W. Walbank, Monarchies and Monarchic Ideas, in: The Cambridge Ancient History. Second Edition. Bd. VII/I (Cambridge, 1984), 62-100.

G. Walser, Audienz beim persischen Großkönig. Zur Geschichte des Hofzeremoniells in der Antike (Zürich, 1965). 
G. Weber, Poesie und Poeten an den Höfen vorbellenistischer Monarchen, in: Klio 74 (1992), 25-77.

G. Weber, Dichtung und höfische Gesellschaft. Die Rezeption von Zeitgeschichte am Hof der ersten drei Ptolemäer (Stuttgart, 1993).

G. Weber, Interaktion, Repräsentation und Herrschaft. Der Königshof im Hellenismus, in: A. Winterling (Hg.), Zwischen, Haus` und ,Staat'. Antike Höfe im Vergleich (München, 1997), 27-71.

G. Weber, Herrscher und Traum in hellenistischer Zeit, in: Archiv für Kulturgeschichte 81 (1999), 1-33.

M. Whitby, Four Notes on Alexander, in: Electrum 8 (2004), 35-47.

H.-U. Wiemer, Alexander der Große (München, 2005).

J. Wiesehöfer, Die ,Freunde und, Wobltäter des Großkönigs, in: Studia Iranica 9 (1980), 7-21.

J. Wiesehöfer, Das antike Persien von 550 v. Chr. bis 650 n. Chr. (München - Zürich, 1994).

G. Wirth, Alexander, Kassander und andere Zeitgenossen. Erwägungen zum Problem der Selbstdarstellung, in: Tyche 4 (1989), 193-220.

G. Wirth, Der Brand von Persepolis. Folgerungen zur Geschichte Alexanders des Großen (Amsterdam, 1993).

M. Zahrnt, Versöhnen oder Spalten? Überlegungen zu Alexanders Verbanntendekret, in: Hermes 123 (2003), 407-432. 